\title{
Genome-wide analysis of mRNA decay patterns during early Drosophila development
}

Stefan Thomsen ${ }^{1,2}$, Simon Anders ${ }^{3,4}$, Sarath Chandra Janga $a^{5,6}$, Wolfgang Huber ${ }^{3,4}$, Claudio R Alonso ${ }^{1,2^{*}}$

\begin{abstract}
Background: The modulation of mRNA levels across tissues and time is key for the establishment and operation of the developmental programs that transform the fertilized egg into a fully formed embryo. Although the developmental mechanisms leading to differential mRNA synthesis are heavily investigated, comparatively little attention is given to the processes of mRNA degradation and how these relate to the molecular programs controlling development.

Results: Here we combine timed collection of Drosophila embryos and unfertilized eggs with genome-wide microarray technology to determine the degradation patterns of all mRNAs present during early fruit fly development. Our work studies the kinetics of mRNA decay, the contributions of maternally and zygotically encoded factors to mRNA degradation, and the ways in which mRNA decay profiles relate to gene function, mRNA localization patterns, translation rates and protein turnover. We also detect cis-regulatory sequences enriched in transcripts with common degradation patterns and propose several proteins and microRNAs as developmental regulators of mRNA decay during early fruit fly development. Finally, we experimentally validate the effects of a subset of cis-regulatory sequences and trans-regulators in vivo.

Conclusions: Our work advances the current understanding of the processes controlling mRNA degradation during early Drosophila development, taking us one step closer to the understanding of mRNA decay processes in all animals. Our data also provide a valuable resource for further experimental and computational studies investigating the process of mRNA decay.
\end{abstract}

\section{Background}

The process of embryonic development, that is, the transformation of the egg into a fully formed embryo, is a heritable feature that relies on the establishment of distinct programs of gene activity in different subregions of the developing organism. Given that the specification and implementation of such gene regulatory programs requires as well as triggers particular spatiotemporal modulations in mRNA levels, the full understanding of the mechanisms regulating mRNA abundance is central to determine how development is molecularly controlled.

In this context, much attention has been focused on the study of transcriptional regulation, leaving the processes that degrade mRNA molecules relatively

\footnotetext{
* Correspondence: C.Alonso@sussex.ac.uk

'John Maynard Smith Building, School of Life Sciences, University of Sussex Falmer, Brighton, BN1 9QG, UK

Full list of author information is available at the end of the article
}

unexplored; this bias does not seem fair given that the abundance of each mRNA species in the embryo is determined not only by the transcriptional rate at which it is produced, but also by the rate of its degradation. Importantly, mRNA degradation rates will ultimately not just dictate the absolute concentration levels of a given mRNA at a given time, but also determine how promptly these levels will react to a change in transcriptional rates: no matter how sensitive and swift a transcriptional switch might be, if the resulting mRNA transcripts have prolonged half-lives, the cell will be indifferent to a change in transcriptional state as long as the transcripts remain stable.

An indication of the potential impact of mRNA degradation can be inferred from the variety of factors controlling mRNA degradation (or decay) rates, including hormones [1,2], viral infections [3], iron levels $[4,5]$, cell cycle progression $[6,7]$ and cell differentiation $[8,9]$. In spite of this, very little is known about the rules 
controlling mRNA decay in a transcript-specific manner, and how such rules interface with the developmental programs encoded in the genome of multi-cellular animals.

We envisage two main reasons for this. Firstly, the rather limited set of examples for which we have both high quality mRNA decay data and precise mapping of decay motifs makes it difficult to infer general principles useful in the identification of general regulatory modules controlling mRNA decay and the factors operating them. Larger datasets would - in principle - allow the systematic search for common features present in transcripts with similar mRNA decay patterns and establish whether functionally related genes share common regulation by mRNA degradation. Secondly, for a successful investigation of mRNA degradation in the physiological environment of animal development, the separate contributions of mRNA synthesis (transcription) and mRNA degradation must be teased apart. This generally implies the need to implement transcriptional shut-off regimes [10-13], which may cause a full spectrum of non-specific effects and developmental arrest, fail to stop transcription uniformly across different tissues [14-17], and, not least, might affect the process of RNA degradation itself by eliminating gene transcription of its regulators.

In this study, we circumvent these problems by carrying out a genome-wide expression analysis during Drosophila melanogaster early development, as this developmental window provides a natural system largely devoid of transcription: developing oocytes pause transcription well before the moment of egg laying [18], and embryos start their transcriptional programs not earlier than 1.5 to $2.0 \mathrm{~h}$ after egg laying (AEL) [19-21]. Therefore, in our experimental design, early modulations in mRNA levels directly reflect mRNA decay. Furthermore, the molecular and cellular events of early Drosophila development (Figure 1a) provide a uniquely characterized framework to address how mRNA decay relates to gene and cell function, as well as the ways in which RNA decay relates to other levels of gene control.

In Drosophila, two machineries of distinct origin - and largely unknown composition - act to remove transcripts of maternal origin from the early Drosophila embryo. One of them, termed the maternal machinery, is entirely driven by maternally encoded factors $[22,23]$ and its activity is triggered by egg activation - a molecular process that prepares the oocyte for embryogenesis [24-26]. The second degradation system is termed the zygotic machinery and becomes active with the onset of zygotic transcription after fertilization. As unfertilized eggs never initiate their own transcriptional programs, all degradation processes active in them will be of maternal nature. Separate maternal and zygotic decay machineries act during early embryonic stages not only in flies but across the bilateria, including nematodes, zebra fish, frogs and mice [27].

A large body of evidence in the literature demonstrates the normal initiation and progression of various post-transcriptional events in unfertilized Drosophila eggs: these include translation [28-31], cytoplasmic polyadenylation [25,32], RNA interference activation [33,34], phosphorylation [29] and, notably, the degradation of several mRNAs [22,35-39]. During the first few hours after egg laying these post-transcriptional events occur with similar kinetics in unfertilized eggs and embryos (see $[25,27,40,41]$ for recent reviews). Interestingly, many of these processes appear associated with fertilization in other model organisms. Due to these considerations, the unfertilized egg system continues to be widely used to study post-transcriptional processes in early Drosophila development [23,28,31,38,39,42-49].

Here we use synchronized samples of Drosophila unfertilized eggs and early embryos in combination with genome-wide microarray technology to study the regulation of global mRNA decay patterns during early fly development. Our analysis led us to (i) determine the diversity in mRNA decay patterns and mRNA decay rates during early fly embryogenesis, (ii) tease apart the maternal and zygotic contributions to mRNA turnover, (iii) establish a relationship between mRNA decay patterns and gene functional classes, (iv) explore how mRNA degradation profiles relate to mRNA localization during early fly development, (v) reveal a coordination of mRNA and protein turnover, (vi) address how particular decay classes relate to target sets for known mRNA decay factors and (vi) identify putative novel cisand trans-regulators and experimentally validate a subset of them. Our work thus makes a significant contribution to the current understanding of the process of mRNA stability control during early animal development.

\section{Results}

Establishing genome-wide mRNA decay profiles during early Drosophila development

Our experimental design compares the transcriptomes derived from Drosophila embryos and unfertilized eggs using a microarray approach. This strategy allows the study of mRNA decay in vivo in the absence of transcriptional inhibition treatments that may affect embryonic development and the processes underlying RNA degradation themselves. Given that during early Drosophila embryogenesis mRNA degradation is controlled by both maternal and zygotic systems, collection of parallel samples from tightly synchronized embryos and unfertilized eggs (Figure 1a) enabled us to tease apart maternally and zygotically controlled mRNA decay processes. 


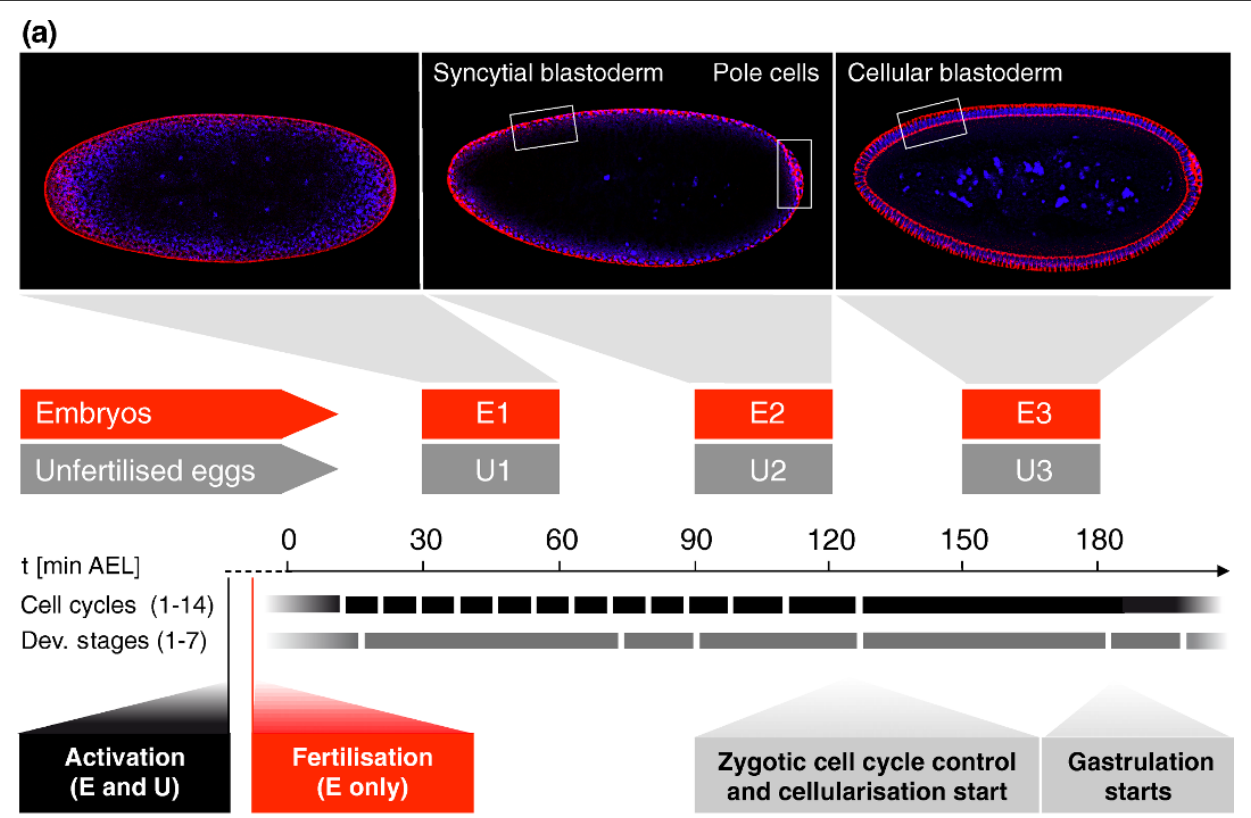

(b)

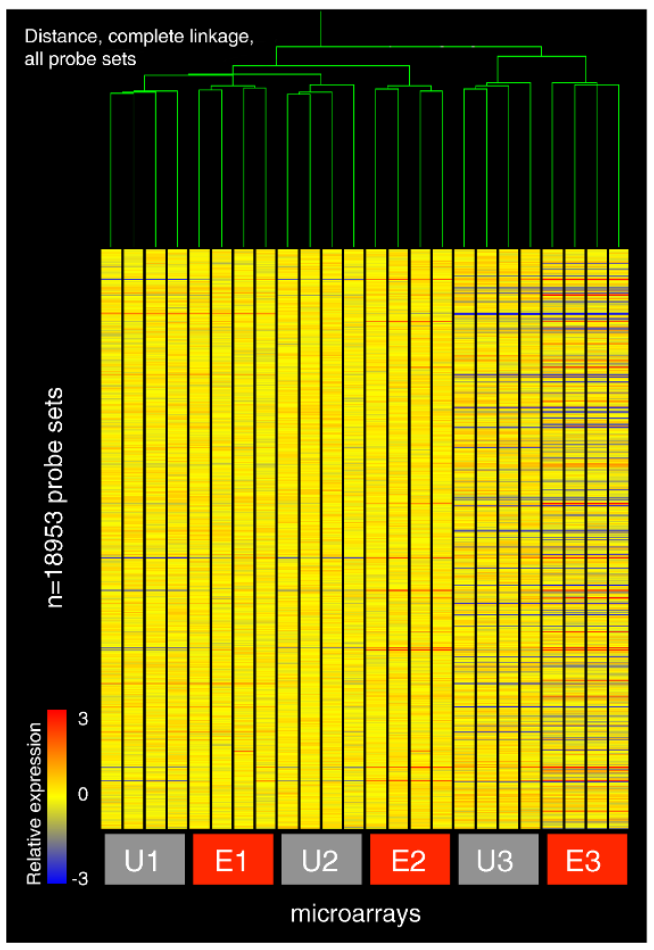

(c)

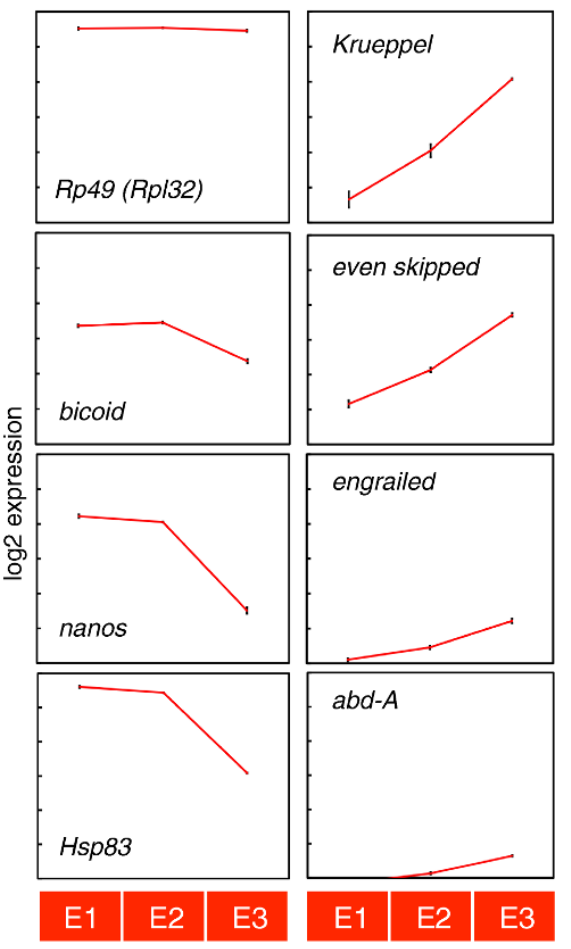

Figure 1 Genome-wide expression profiles in early Drosophila embryos and unfertilized eggs. (a) Microarray time course. Experimental design: sampling intervals, morphological features of embryos, cell cycles (black bars), developmental stages after Hartenstein [111] (grey bars) and hallmarks of early fly development (grey boxes) are indicated. Confocal embryo images: DAPI/FITC-phalloidin stain to highlight cell nuclei (blue) and cell cortices (actin, red). Four replicate samples were analyzed for each treatment. (b) Microarray data quality assessment. Hierarchical clustering (Pearson correlation distance) grouped 24 microarrays ( $x$-axis) into 6 replicate groups (see (a)). Expression levels for approximately 19,000 probe sets (y-axis) are shown in relation to median expression for each probe set across all microarrays. (c) Sample microarray expression profiles. Median log2 expression of four biological replicates; 1 Unit = log2 fold-change 1; error bars represent standard error of the mean over replicates. 
We began our study sampling mRNAs from three time points during early embryogenesis (30 to $60 \mathrm{~min}$ utes (E1), 90 to 120 minutes (E2), and 150 to $180 \mathrm{~min}$ utes (E3) AEL) as well as matching samples from unfertilized eggs (U1, U2 and U3) (Figure 1a). Both embryos and unfertilized eggs were wild type (Oregon Red). Four biological replicates were collected from each time point and analyzed using Drosophila Genome 2.0 GeneChips. We used Bioconductor software to preprocess and assess the quality of our data. Hierarchical clustering showed that biological replicates always formed tight clusters, reflecting the quality and reproducibility of our methods for sample isolation and analysis (Figure 1b); further quality assessments using spatial and numeric diagnostics corroborated that our microarray data were of high quality (Supplementary Figure 1 in Additional file 1).

Previous microarray expression analyses [46,50,51] and studies measuring incorporation of radioactively labeled monomers into nucleic acids [30,52-54] had reported undetectable rates of RNA decay or synthesis prior to our first time point (30 to 60 minutes, U1 + E1). To further confirm this, we investigated the presence of early RNA decay and synthesis by comparing expression levels in U1 and E1 samples to stage 14 egg chambers; the latter comprise both the unactivated oocyte and somatic follicle cells (Supplementary Figure 3 and Supplementary materials and methods in Additional file 1). This analysis confirmed the absence of significant transcription and RNA decay prior to our first time point. Considering the unavoidable presence of follicle cell transcripts in stage 14 egg chamber RNA samples (Supplementary Figure 3b, $d$ in Additional file 1), the finding of identical expression levels in stage $14 \mathrm{egg}$ chambers and U1 samples led us to choose the latter as our reference time point zero for subsequent analyses.

Normalized transcript expression levels were independently validated by a comprehensive quantitative PCR experiment monitoring the expression of 24 genes chosen to represent the wide spectrum of expression patterns seen in our dataset (Supplementary Figure 2a in Additional file 1). Furthermore, our microarray data profiles were coherent with previous degradation data for specific mRNAs (for example, rp49, bicoid, nanos, Hsp83) [22,55] and consistent with the expected temporal sequence of expression for the Drosophila segmentation cascade genes in late embryonic samples (Krueppel, even-skipped, engrailed, abd-A) (Figure 1c).

Given that in our system modulations of transcript abundance reflect the course of mRNA decay processes, once the quality of our microarray experiment was confirmed, we went on to examine the spectrum of mRNA decay profiles in our biological samples.
To determine the diversity of mRNA decay patterns in the embryonic samples, we first identified all unstable transcripts in embryo collections with a significant reduction between E1 and E3 and performed a hierarchical clustering of their expression profiles. We show the behavior of several sub-clusters of mRNAs with comparable initial expression levels in Figure 2a. We observed a wide diversity in net decay amplitudes between E1 and E3 (Figure 1a) as well as in the particular temporal profiles of individual transcripts. We note that within the sampled period some transcripts experienced only a modest net decay while others demonstrated a severe reduction in concentration; in addition, some mRNAs showed significant degradation between E1 and E2 (early decay; Figure 2a(i,iii,iv)) while others were initially stable and then decayed swiftly between E2 and E3 (late decay; Figure 2a(ii)).

These initial observations prompted us to quantify net decay values and to explore early and late decay contributions to individual decay profiles genome-wide (Figure $2 b, c)$. Note that decay values reported here are differences of $\log 2$ expression values; hence, they represent the $\log 2$ change-folds (or ratios of expression) between the respective time points. For instance, a net decay of -1 is equivalent to a decrease of $50 \%$ in transcript signal. Studying the distribution of global net decay values in embryos for all unstable transcripts (Figure 2b), we found a maximum net decay of -5.8 , equivalent to a reduction to less than $2 \%$ of the initial expression value (Figure 2b, note lower whisker in the boxplot) and a median net decay of -1.3 , equivalent to a reduction to approximately $40 \%$. The majority of probes $(75 \%)$ detecting destabilized transcripts showed a significant reduction in mRNA abundance of at least 35\% (log2 change-fold -0.6; Figure 2b, boxplot upper percentile).

To determine the proportion of transcripts following an early or late mode of degradation, we then partitioned net decay values into early and late decay and plotted them against each other (Figure 2c). This analysis indicated that while hundreds of transcripts experience significant early decay between 0.5 and $2 \mathrm{~h}$ AEL, most mRNAs were degraded late between 1.5 and $3 \mathrm{~h}$ AEL.

\section{Resolving maternal and zygotic contributions to mRNA decay}

Having analyzed the salient features of global mRNA decay profiles in embryos, we turned to study the factors controlling global embryonic mRNA behavior. For this, we made use of to the microarray data derived from unfertilized eggs (Figure 1a): to investigate the contributions of the maternal and zygotic machineries to mRNA degradation, we compared the mRNA decay patterns obtained in embryos with those recovered from 


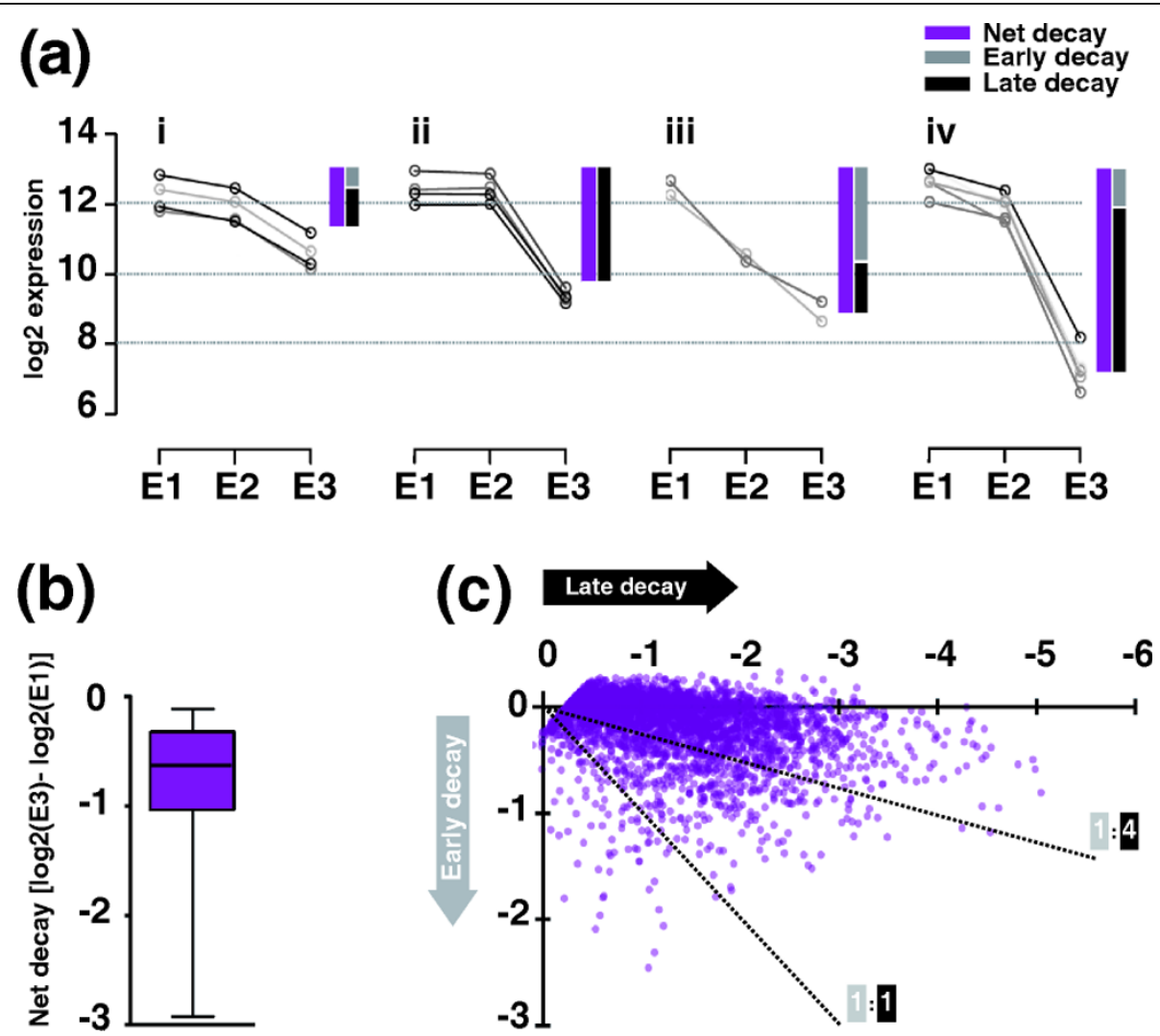

Figure 2 Diversity of mRNA decay patterns in Drosophila embryos. (a) Clusters of mRNA decay profiles in early embryos (E1, E2 and E3 (Figure 1a)). We show a selection of profiles with increasing net decay amplitudes (purple bar, filled) and differential contributions of early and late decay (grey and black bars, respectively). (b) Global distribution of net mRNA decay (box plot with median and lower/upper quartile, whiskers from minimum to maximum); we considered all probe sets where E3 is significantly lower than E1 (3,658 probe sets; Figure 1a). (c) Net decay partitioned into early and late decay: major decay events took place late between 2 and $3 \mathrm{~h}$ AEL (note high density of points close to $x$ axis); a subset of transcripts showed early decay between 1 and2 $\mathrm{h}$ AEL. Dotted lines indicate the ratio of early and late decay (1:1 or 1:4).

unfertilized eggs, a system solely relying on the maternal machinery. We reasoned that for each mRNA species in the embryo, the concentration of its mRNA $X$ at a particular time $t$ AEL is determined by the following relationship:

$$
\text { (Embryos) } X(t)=X_{M}+\Delta X_{T}(t)-\Delta X_{M D}(t)-\Delta X_{Z D}(t)
$$

Here, $X_{M}$ is the initial concentration of mRNA that is maternally provided during oogenesis, $\Delta X_{T}$ is the increase in concentration of mRNA as provided by embryonic transcription, $\Delta X_{M D}$ represents the decrease in concentration as a consequence of mRNA decay caused by maternal factors, and $\Delta X_{Z D}$ is the decrease in concentration caused by zygotically encoded mRNA decay factors. We summarize the sign (+/-) of the different contributions to mRNA levels, their occurrence and respective timing in embryos and unfertilized eggs in Figure 3a (top left panel). Given that in unfertilized eggs all contributions relying on de novo mRNA synthesis are null, the concentration of mRNA $X_{s}$ at time $t$ AEL is dictated by the simplified relationship:

$$
\text { (Unfertilized eggs) } \quad X(t)=X_{M}-\Delta X_{M D}(t)
$$

From this framework we considered that the integration of mRNA expression information from embryos and unfertilized eggs at different time points would make it possible to tease apart the contributions of maternal and zygotic decay to individual mRNA species. Given that our data (Supplementary Figure 3b in Additional file 1) as well as previous microarray results (see above, and $[46,50,51]$ ) demonstrated that global RNA levels in stage 14 oocytes and early unfertilized egg (U1) are comparable, we assumed that mRNA concentrations in the latter should be informing us about the levels of maternal provision $X_{M}$ for each mRNA species. Therefore:

$$
\text { ( Early unfertilized eggs, } U 1) \quad X(U 1)=X_{M}
$$



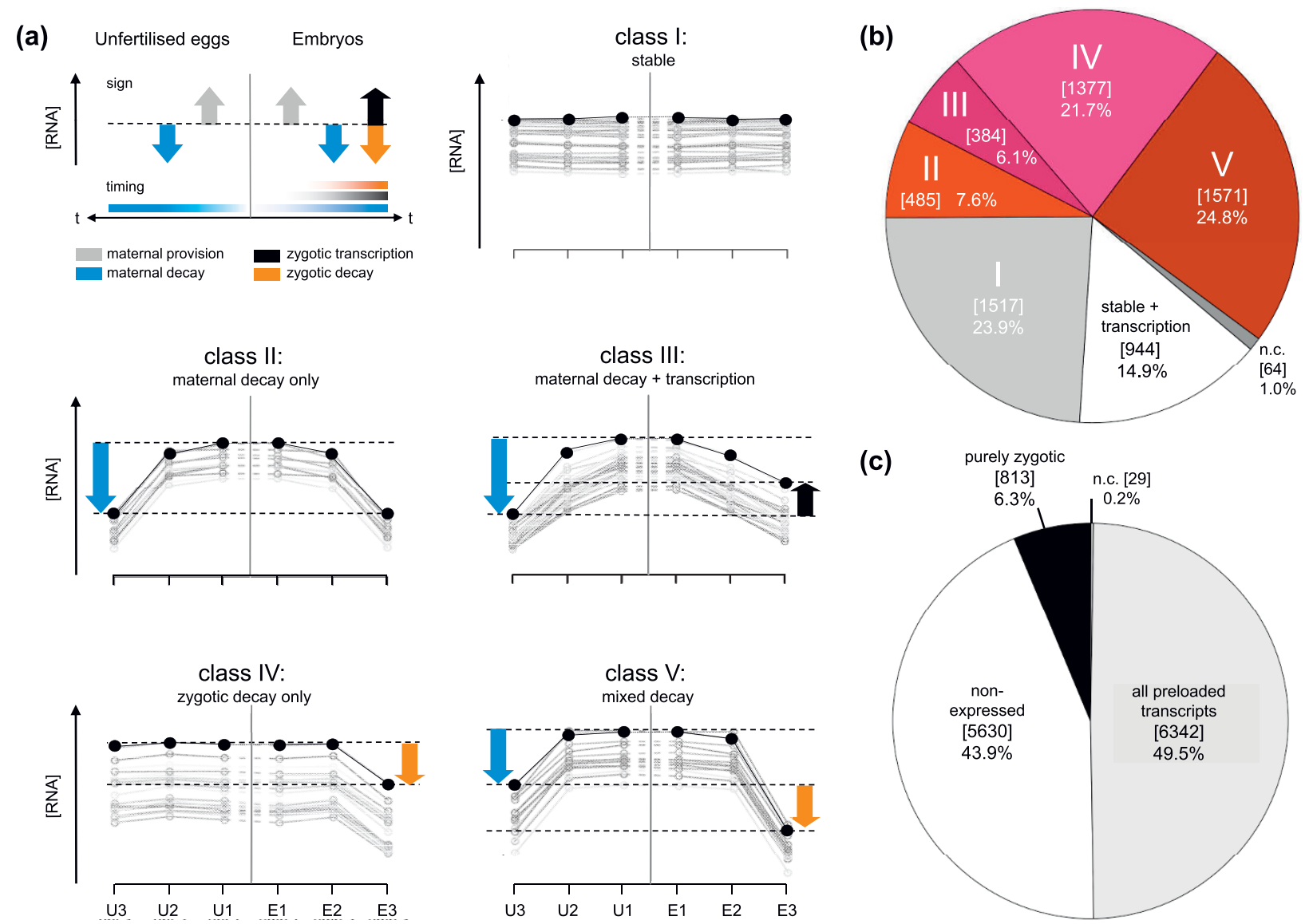

Figure 3 Classification of mRNA expression profiles in early embryos. (a) mRNA pools in embryos are shaped by (i) maternal provision, (ii) transcription, (iii) maternal decay activities and (iv) zygotic decay activities. The sign (+/-) of these contributions to RNA levels and their differential timing is indicated on a time scale for both unfertilized eggs (centre to left, U1 to U3) and embryos (centre to right, E1 to E3). mRNA expression profiles were classified into five major stability classes; clusters of prototypical example profiles are shown for classes I to V. (b) Preloaded, maternal transcriptome: proportions and gene numbers (in square brackets) for classes I to $V$ representing a total of 6,342 genes. (c) Transcriptome of the early embryo: proportion and gene numbers of non-expressed, purely transcribed and maternally provided mRNAs representing a total of 12,814 unique genes. n.c., non-classified and complex patterns.

Analysis of expression levels in unfertilized egg samples U1, U2 and U3 over time (Figure 1a) allowed us to determine the effects of maternal decay factors $\left(\Delta X_{M D}\right)$ on each mRNA species present in these samples (Equation 2) and the comparison of mRNA levels in embryos and unfertilized eggs enabled us to detect mRNA modulations due to zygotic decay or transcriptional patterns $\left(\Delta X_{M D}, \Delta X_{Z D}\right)$. We note that our system allowed us to detect the dominant or net effect of transcription and zygotic decay where they occur concomitantly (Figure 3a, top-left panel; see Additional file 1 for discussion). This classification allowed us to establish five major decay classes: stable (class I); exclusively maternally degraded (class II); maternally degraded and transcribed by the embryo (class III); exclusively zygotically degraded (class IV); and both maternally and zygotically degraded (mixed decay class V) (Figure 3a). In addition, we detected mRNAs that are transcribed by the embryo, either anew (purely zygotic) or as an addition to a stable, preloaded pool (stable + transcription) (Figure $3 \mathrm{~b}, \mathrm{c}$ ). The classifications for all probe sets have been deposited in the ArrayExpress Database (see below).

We then determined the fraction of the transcriptome represented in each mRNA class (Figure 3b). Our quantification revealed that transcripts of the majority of genes present in the embryo (60\%) suffer degradation during the first $3 \mathrm{~h}$ of development (classes II to $\mathrm{V}$, 3,817 genes). Of these, more than one-third were targeted by both maternal and zygotic decay factors (class $\mathrm{V}, 24.8 \%, 1,571$ genes). There were 1,377 mRNAs targeted by exclusive zygotic decay activities (class IV, 21.7\%), while 485 mRNAs suffered exclusive maternal decay (class II, 7.6\%). Another 384 transcripts were maternally degraded but also transcribed by the embryo 
(class III, 6.1\%). All in all we found that $40 \%$ of preloaded transcripts were targeted by maternal decay activities (classes II, III, V), a fraction much higher than previously estimated [43]. We also noted that $45 \%$ of transcripts in the embryo were targeted by zygotic decay activities (classes IV, V).

We also detected wide overlaps of maternal provision, decay and transcription - as $>20 \%$ of all maternally provided mRNAs were supplemented by transcription class III, stable + transcription) - and that mRNAs for $50 \%$ of the Drosophila genes were preloaded onto the egg during oogenesis (Figure 3c); these findings are in good agreement with previous estimates $[28,43,46,56]$.

\section{Maternal decay activities in early embryos are fast and efficient}

Having established the proportions of the transcriptome that belong to each decay category, we explored the kinetic features of decay processes within each class, focusing on net decay values and half-lives.

We calculated net decay values as the difference between $\log 2$ expression values of late time points (U3 or E3) and early unfertilized eggs (U1) (Figure 4a) and show the distributions of net decay values in different classes (Figure $4 \mathrm{~b}$ ). We also estimated individual transcript half-lives from expression levels in late embryos or unfertilized eggs assuming an exponential decay model, which we applied to samples taken from $t_{2}$ and $t_{3}$ of the respective time series (U2 and U3 for classes II and III; E2 and E3 for classes IV and V; Figure 4a). We selected this model and temporal frame for our calculations because global RNA decay studies had shown a good fit of data to exponential decay models $[13,16,57,58]$ and most decay events occur between 2 and $3 \mathrm{~h}$ AEL (Figure 2c), respectively. Inspection of thousands of decay profiles suggested that mRNA decay patterns generally exhibited a lag phase followed by a decay phase of variable lengths (Figures 2a, c, 3a and 4a, and data not shown). Ideally, these decay curves would be mathematically modeled as a concatenation of a lagphase transitioning into an exponential decay curve. However, fitting our data to this type of model would have required more time points than the ones we had available. We derived transcript half-life estimates for 3,817 mRNAs and report the distribution of half-lives in all decay classes (Figure 4c). It should be noted that half-lives and net-decay values reported here are lower bound estimates (see Additional file 1 for discussion).

We saw that transcripts with maximum net decay and lowest half-lives belonged to classes with maternal decay contributions (II, III, V); for instance, degradation in these classes could lead to more than $97 \%$ reduction of mRNA levels (net decay less than -5; Figures 4b, c, minimum values of lower whiskers). Median decay values for classes II to $\mathrm{V}$ were $-1.2,-2.4,-0.4$ and -1.7 , translating into average mRNA level reductions of approximately $57 \%, 80 \%, 25 \%$ and $70 \%$. For the mixed decay class $(\mathrm{V})$ we saw that the median maternal contribution was significantly higher than the median zygotic contribution (Supplementary Figure 6a in Additional file 1 ) and that the maternal decay contribution outweighed the zygotic one for the majority of mRNAs (64\%; Supplementary Figure $6 \mathrm{~b}$ in Additional file 1). Median halflives for classes II to $\mathrm{V}$ are 64, 31, 133 and 38 minutes, respectively. Net decay and half-lives for selected mRNAs representing a wide range of kinetic profiles are shown in Figure 4d, and the 50 genes with the highest net decay in classes II to V are presented in Supplementary Table 2 in Additional file 1.

We also explored the origin of early and late mRNA decay patterns detected in embryos (Figure 2c). Maternal decay activity regulators are preloaded onto the egg and, unlike zygotic activities, are independent of de novo transcription in the embryo. In line with these features, we found that early decay is detectable only in stability classes with maternal decay contributions (II, III, V) while exclusively zygotic decay (class IV) is generally late (Figure 4e).

To explore the continuity of maternal and zygotic decay activities beyond the time frame of our time series (Figure 1a), we turned to data from a recent expression study in embryos that provide high temporal resolution during gastrulation stages [59] (Supplementary Figure 8 in Additional file 1). Following up the degradation of hundreds of transcripts with exclusively maternal (class II), exclusively zygotic (class IV) or mixed decay patterns (class V), we observe that degradation continues beyond $3 \mathrm{~h}$ AEL only for mRNAs in zygotic decay classes (IV and V) (see Additional file 1 for detailed analysis). This suggests that maternal decay events are, overall, completed by the onset of gastrulation while zygotic decay events continue throughout this developmental phase.

Taken together, we conclude that the dual action of maternal and zygotic decay activities (class V) leads to more pronounced decay patterns than maternal or zygotic decay alone (classes II and IV), suggesting a lack of redundancy between these machineries. We also note that most severe decay patterns were mediated by maternal decay activities acting on preloaded mRNAs with parallel transcription (class III).

\section{Relating mRNA decay to gene function}

Studies in bacterial, yeast and mammalian cell culture systems had shown that rates of transcript decay can vary significantly across different functional categories and that messages encoding components of multi-protein complexes decay at similar rates $[12,16,60-65]$. To establish how mRNA stability relates to gene function in 
(a)

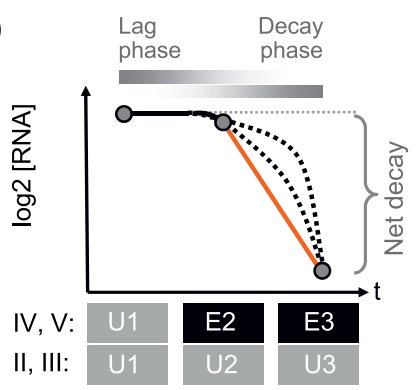

(b)
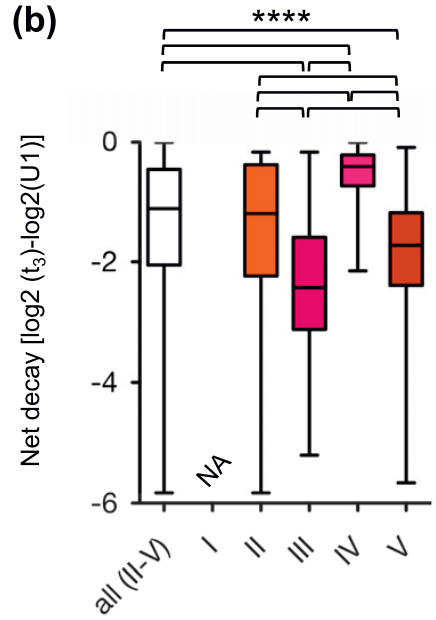

(c)
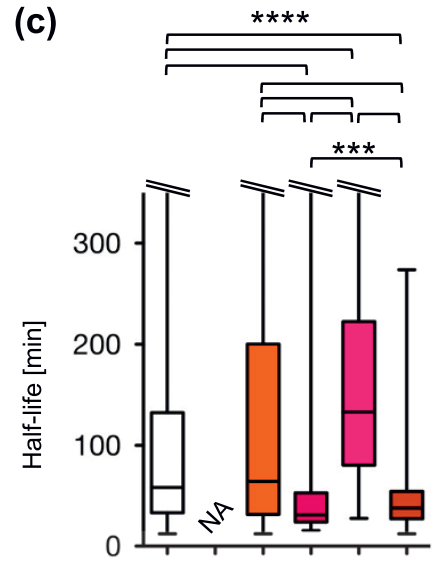

งก) (d)

\begin{tabular}{c|c|c|c} 
Gene & Class & $\begin{array}{c}\text { Net decay } \\
\text { [log2 changefold] }\end{array}$ & $\begin{array}{c}\text { Half-life } \\
\text { [min] }\end{array}$ \\
\hline Cyp6a19 & II & -5.8 & 12 \\
exu & V & -5.7 & 13 \\
Gapdh2 & V & -4.8 & 14 \\
BicC & V & -4.6 & 14 \\
orb & V & -4.0 & 16 \\
Pgi & III & -4.6 & 16 \\
nod & II & -3.4 & 19 \\
osk & V & -3.0 & 20 \\
cort & II & -3.9 & 23 \\
nos & V & -2.8 & 24 \\
gnu & V & -2.6 & 25 \\
png & V & -2.9 & 25 \\
otu & V & -2.4 & 25 \\
Hsp83 & V & -2.5 & 25 \\
spz & V & -2.2 & 26 \\
elF-4E & V & -1.8 & 34 \\
Sara & V & -1.8 & 35 \\
btz & V & -1.5 & 40 \\
twin & V & -1.3 & 43 \\
stg & V & -1.3 & 45 \\
hb[mat] & III & -1.4 & 51 \\
bcd & IV & -1.0 & 55 \\
tral & V & -0.9 & 65 \\
spir & IV & -0.8 & 66 \\
plu & IV & -0.9 & 66 \\
smg & IV & -0.8 & 69 \\
CycB & IV & -0.8 & 76 \\
tor & IV & -0.6 & 99 \\
nudC & V & -0.7 & 115 \\
hk & IV & -0.6 & 143 \\
cact & III & -0.3 & 186 \\
Dys & IV & -0.2 & 202 \\
& & &
\end{tabular}

(e)

Late decay

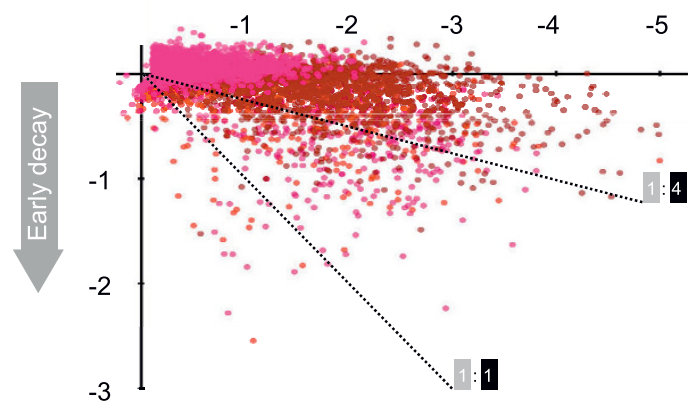

Figure 4 Kinetics of maternal and zygotic RNA decay activities. (a) Quantification of mRNA decay by measuring global net decay amplitudes and estimating mRNA half-lives. The red line represents the assumed exponential decay between $t_{2}$ and $t_{3}$; dotted lines represents the possible non-exponential decay kinetics. (b) Distribution of net decay amplitudes in classes I to V. (c) Distribution of transcript half-lives in classes I to V. Significant differences in medians are indicated by brackets (pairwise comparisons, two-tailed Mann-Whitney test): ${ }^{* * *} P \leq 0.001$; ${ }^{* * * *} P<0.0001$. All box plots are shown with median and lower/upper quartile, whiskers from minimum to maximum. (d) mRNA decay rates and half-lives for selected genes. (e) Timing of mRNA decay: early versus late decay in classes II to V. Dotted lines indicate the ratio of early and late decay $(1: 1$ or 1:4). Class labels and color codes are as in Figure $3 \mathrm{~b}$. 
the physiological context of early fly development, we identified the cellular components, gene functions and biological processes associated with unstable or stable mRNAs using Gene Ontology (GO; Tables 1 and 2).

This analysis revealed that the many short-lived transcripts show associations with chromatin and the replication machinery. The specific gene functional and biological themes associated with unstable mRNAs were (i) cell cycle control, (ii) DNA metabolism, replication and repair, (iii) establishment of localization in cells, and (iv) non-coding RNA metabolic processes (Table 1). This last finding prompted us to explore the stabilities of transcripts encoding products related to mRNA destabilization and the biochemistry of small RNAs (Table 3). We found, indeed, that transcripts for key players of the microRNA (miRNA) (dicer-1), the piwiinteracting RNA (piRNA) (aubergine, piwi) and the small interfering RNA (siRNA) pathway (dicer-2, $r 2 d 2$, $v i g$, and so on) suffered significant degradation during the first $3 \mathrm{~h}$ of development. We also noted significant mRNA decay for genes of the nonsense-mediated mRNA decay pathway and generic deadenylation, decapping and decay factors. In addition, we found that mRNAs of cortex (cort), grauzone (grau), wispy (wisp, CG15737), pan gu (png), plutonium (plu) and giant nuclei (gnu), all of which are required for maternal mRNA decay activities [39], suffered considerable degradation (see also Figure 4d). These findings were consistent with a need to readjust expression levels of these regulators once the zygotic genome resumes control over the developmental program of the embryo.

Stable mRNAs showed strong associations with ribosomes and ribonucleoprotein complexes (Table 2). Accordingly, enriched gene functions and biological processes related largely to structural ribosome constituents and various RNA transactions (mRNA binding, RNA metabolic process, RNA processing). Further themes related to translation control, posttranslational modifications and energy allocation (electron transport chain, oxidative phosphorylation). These observations are consistent with a constant requirement for these processes throughout early development.

\section{mRNA decay is linked to posterior mRNA localization patterns}

Our functional analysis of unstable mRNAs suggested a link between mRNA decay and the establishment of localization in the developing embryo (Table 1). To explore the way in which mRNA decay may contribute to localization and developmental patterning in the early embryo, we studied the connections between mRNA degradation and localization in more detail.

To do this, we used the Fly-FISH database $[66,67]$, which provides spatial information for more than 3,000
mRNAs over different stages of embryogenesis (Supplementary Figure 8 in Additional file 1) at the whole embryo and subcellular levels (Figure 5).

We first asked whether genes with particular localization patterns are overrepresented or depleted in any of our transcript classes. Figure 5 shows respective enrichment and depletion patterns for 26 localization terms as a heatmap sorted by general themes: (i) anterior localization, (ii) localization at the posterior of the embryo and in pole cells, (iii) localization patterns related to nuclear and transcriptional patterns, and (iv) degradation patterns. This analysis revealed strong correlations between mRNA decay and localization.

Localization terms related to posterior localization were highly enriched in several mRNA decay classes (posterior localization, pole buds, RNA islands, pole cell localization, pole cell enrichment and pole plasm; see Supplementary Table 4 in Additional file 1 for a full list of unstable mRNAs in these categories). We saw strongest enrichments in decay classes with exclusively zygotic or mixed decay patterns (classes IV and V); note, for instance, the strong enrichment patterns for the localization term 'pole cell localization' in decay classes IV and V (Supplementary Figure 7 in Additional file 1). The links between posterior mRNA localization and mRNA decay are further validated by the fact that unstable transcripts of decay classes II, IV and V are significantly depleted for the terms 'pole plasm excluded' and 'pole cell exclusion' (Supplementary Figure 7 in Additional file 1). In summary, we observed a strong positive correlation between mRNA decay and posterior mRNA localization patterns.

Four out of five genes listed in Fly-FISH with anterior localization ( $b c d, C y c B$, lok, milt, asp) showed zygotic or mixed decay patterns (see classification data deposited at ArrayExpress); however, due to the low number of genes, this observation was not considered significant at a $10 \%$ false discovery rate. Nuclear and transcriptional patterns (theme (iii)) were exclusively enriched in classes with transcription (class III, purely zygotic, stable + transcription) while degradation-related expression patterns (theme (iv)) were enriched in decay classes. Taken together, Fly-FISH mRNA annotations are consistent with our own mRNA classification and provide independent support for its validity.

\section{mRNA and protein turnover are coordinated in early embryos}

Ultimately, most protein-encoding mRNAs exert their function at the protein level. Having established that a large proportion of the preloaded mRNA pool is being removed from the early embryo by RNA decay, we wondered whether these changes in RNA levels - perhaps reflecting a need to reduce or eliminate the expression 
Table 1 Relating mRNA decay to gene function

\begin{tabular}{ll}
\hline GO term & $P$-value \\
\hline Cellular component & \\
Replication fork & $2.38 \mathrm{E}-06$ \\
Nuclear chromosome & $5.18 \mathrm{E}-05$ \\
Nuclear chromosome part & $6.91 \mathrm{E}-05$ \\
Chromosome & $4.95 \mathrm{E}-04$ \\
Microtubule organizing centre part & $1.78 \mathrm{E}-03$ \\
Replisome & $1.96 \mathrm{E}-03$ \\
Nuclear replisome & $1.96 \mathrm{E}-03$ \\
Nuclear replication fork & $1.96 \mathrm{E}-03$ \\
Endoplasmic reticulum membrane & $2.77 \mathrm{E}-03$ \\
Nuclear envelope-endoplasmic reticulum network & $3.35 \mathrm{E}-03$ \\
Endomembrane system & $4.43 \mathrm{E}-03$ \\
Rough endoplasmic reticulum membrane & $9.11 \mathrm{E}-03$
\end{tabular}

Gene function

Rough endoplasmic reticulum membrane 9.11E-03

Transferase activity $\quad 4.13 \mathrm{E}-11$

Lipid binding 4.88E-06

Zinc ion binding 1.37E-05

Cofactor binding 2.37E-05

Nucleoside-triphosphatase activity 3.97E-05

DNA-dependent ATPase activity 4.66E-05

Pyrophosphatase activity $\quad$ 8.22E-05

Metal ion binding $\quad$ 8.64E-05

DNA-directed DNA polymerase activity 1.09E-04

Cation binding 1.14E-04

lon binding 1.18E-04

Aminoacyl-tRNA ligase activity $\quad 1.18 \mathrm{E}-04$

Ligase activity, forming aminoacyl-tRNA and related $\quad 1.18 \mathrm{E}-04$

compounds

Ligase activity, forming carbon-oxygen bonds 1.18E-04

Hydrolase activity, acting on acid anhydrides, in $\quad 1.21 \mathrm{E}-04$

phosphorus-containing anhydrides

Transferase activity, transferring phosphorus- 2.20E-04

containing groups

Hydrolase activity, acting on acid anhydrides 2.40E-04

DNA polymerase activity 2.94E-04

DNA binding $\quad 3.42 \mathrm{E}-04$

Ligase activity 6.84E-04

Transition metal ion binding $\quad 1.28 \mathrm{E}-03$

ATPase activity 2.13E-03

Nucleotidyltransferase activity $\quad 3.77 \mathrm{E}-03$

Phosphoinositide binding 6.49E-03

DNA helicase activity 6.87E-03

Coenzyme binding $\quad$ 7.56E-03

Biological process

DNA metabolic process 3.34E-13

Cellular ketone metabolic process $\quad 1.19 \mathrm{E}-11$

Oxoacid metabolic process $3.59 \mathrm{E}-11$

Organic acid metabolic process $\quad 3.59 \mathrm{E}-11$

Carboxylic acid metabolic process $\quad 3.59 \mathrm{E}-11$
Table 1 Relating mRNA decay to gene function (Continued)

\begin{tabular}{|c|c|}
\hline DNA replication & $7.52 \mathrm{E}-10$ \\
\hline Macromolecule localization & $8.71 \mathrm{E}-07$ \\
\hline Cellular localization & $1.35 \mathrm{E}-06$ \\
\hline Cellular response to stress & 1.44E-06 \\
\hline Cellular response to stimulus & 2.09E-06 \\
\hline Cellular amine metabolic process & 3.25E-06 \\
\hline Cellular amino acid metabolic process & $3.25 \mathrm{E}-06$ \\
\hline Cellular macromolecule localization & 8.09E-06 \\
\hline Cellular response to DNA damage stimulus & 1.00E-05 \\
\hline Response to DNA damage stimulus & $2.21 \mathrm{E}-05$ \\
\hline Response to stress & 7.37E-05 \\
\hline Cellular carbohydrate metabolic process & $1.20 \mathrm{E}-04$ \\
\hline DNA repair & 1.43E-04 \\
\hline Cofactor metabolic process & 2.09E-04 \\
\hline Cellular amino acid and derivative metabolic process & $2.14 \mathrm{E}-04$ \\
\hline Localization & 2.24E-04 \\
\hline Establishment of protein localization & $2.52 \mathrm{E}-04$ \\
\hline Protein transport & $3.45 \mathrm{E}-04$ \\
\hline Regulation of cellular component organization & 3.67E-04 \\
\hline Cellular catabolic process & 3.77E-04 \\
\hline Establishment of localization & 8.90E-04 \\
\hline tRNA aminoacylation for protein translation & 1.69E-03 \\
\hline tRNA aminoacylation & 1.69E-03 \\
\hline Regulation of cell cycle & 1.69E-03 \\
\hline Amino acid activation & $2.11 \mathrm{E}-03$ \\
\hline Organelle fission & 2.67E-03 \\
\hline Establishment of localization in cell & $3.75 \mathrm{E}-03$ \\
\hline DNA-dependent DNA replication & $3.84 \mathrm{E}-03$ \\
\hline ncRNA metabolic process & $5.46 \mathrm{E}-03$ \\
\hline Pyruvate metabolic process & $5.86 \mathrm{E}-03$ \\
\hline Transport & 7.27E-03 \\
\hline Monocarboxylic acid metabolic process & $8.38 \mathrm{E}-03$ \\
\hline Anatomical structure formation & 8.67E-03 \\
\hline
\end{tabular}

Gene ontology (GO) analysis for the top 1,000 unstable mRNAs in early embryos using GO::TermFinder. We report significant GO terms and associated $P$-values unique to unstable mRNAs. Cutoff $P$-value $=0.01$.

of certain gene products - were mirrored at the level of protein production or turnover.

To do this comparison between RNA and protein levels, we turned to two recent genome-wide studies addressing translation rates and protein level changes in early Drosophila embryos. In the first study the authors used a ribosomal profiling approach to identify translationally active or silent mRNAs in embryos at 0 to $2 \mathrm{~h}$ AEL [68]; the second study investigated protein levels in embryos at 0 to 90 minutes AEL and 180 to $270 \mathrm{~min}-$ utes AEL [69] (see Supplemental Figure 8 in Additional file 1). Having extracted the respective gene lists from these studies, we performed an enrichment analyses for actively translated and translationally silent mRNAs as well as up- and down-regulated proteins within our transcript classes (Figure 6). 
Table 2 Relating mRNA stability to gene function GO term $P$-value

Cellular component

Ribosomal subunit

2.99E-53

Cytosolic ribosome

$6.21 \mathrm{E}-53$

Ribosome

$2.54 \mathrm{E}-47$

Cytosolic part

$6.46 \mathrm{E}-44$

Ribonucleoprotein complex

5.29E-41

Large ribosomal subunit

$1.75 E-33$

Cytosolic large ribosomal subunit

$5.77 \mathrm{E}-33$

Small ribosomal subunit

1.10E-18

Cytosolic small ribosomal subunit

Cytosol

Nuclear part

Organelle lumen

Intracellular organelle lumen

Mitochondrial ribosome

Organellar ribosome

Respiratory chain

Mitochondrial respiratory chain

Mitochondrial membrane part

Organelle envelope

Envelope

Mitochondrial large ribosomal subunit

Organellar large ribosomal subunit

Mitochondrial membrane

Mitochondrial envelope

Organelle inner membrane

Mitochondrial inner membrane

Nuclear lumen

Gene function

Structural constituent of ribosome

3.24E-49

Structural molecule activity

$5.11 \mathrm{E}-28$

mRNA binding

Enzyme binding

General RNA polymerase II transcription factor activity

Translation regulator activity

Translation factor activity, nucleic acid binding

Biological process

Cellular protein metabolic process

$1.74 \mathrm{E}-32$

Mitotic spindle elongation

9.89E-29

Spindle elongation

2.47E-28

Gene expression

2.44E-27

$3.35 \mathrm{E}-23$

3.40E-23

Cellular macromolecule biosynthetic process

Biopolymer biosynthetic process
Table 2 Relating mRNA stability to gene function (Continued)

\begin{tabular}{|c|c|}
\hline Macromolecule biosynthetic process & $4.56 \mathrm{E}-23$ \\
\hline Translation & $2.21 \mathrm{E}-19$ \\
\hline Protein metabolic process & $3.94 \mathrm{E}-18$ \\
\hline RNA metabolic process & $6.51 \mathrm{E}-09$ \\
\hline Biopolymer modification & 2.33E-08 \\
\hline Protein modification process & 3.57E-08 \\
\hline Phosphorylation & 4.52E-07 \\
\hline Regulation of metabolic process & $5.02 \mathrm{E}-07$ \\
\hline Phosphorus metabolic process & 5.34E-07 \\
\hline Phosphate metabolic process & 5.34E-07 \\
\hline Post-translational protein modification & $1.51 \mathrm{E}-06$ \\
\hline Regulation of macromolecule metabolic process & 2.33E-06 \\
\hline $\begin{array}{l}\text { Mitochondrial ATP synthesis coupled electron } \\
\text { transport }\end{array}$ & $6.52 \mathrm{E}-06$ \\
\hline ATP synthesis coupled electron transport & 2.63E-05 \\
\hline Membrane invagination & 3.03E-05 \\
\hline Endocytosis & 3.03E-05 \\
\hline Electron transport chain & 3.67E-05 \\
\hline Regulation of primary metabolic process & 4.98E-05 \\
\hline Oxidative phosphorylation & 5.61E-05 \\
\hline Respiratory electron transport chain & $6.89 \mathrm{E}-05$ \\
\hline RNA processing & 7.50E-05 \\
\hline Regulation of cellular metabolic process & $9.72 \mathrm{E}-05$ \\
\hline Macromolecular complex assembly & 1.33E-04 \\
\hline Macromolecular complex subunit organization & 2.36E-04 \\
\hline Cellular macromolecular complex assembly & 2.42E-04 \\
\hline Membrane organization & 4.64E-04 \\
\hline $\begin{array}{l}\text { Cellular macromolecular complex subunit } \\
\text { organization }\end{array}$ & 5.06E-04 \\
\hline Regulation of cellular process & $5.24 \mathrm{E}-04$ \\
\hline Regulation of gene expression & $6.81 \mathrm{E}-04$ \\
\hline Cellular component assembly & 8.16E-04 \\
\hline Cellular respiration & $1.78 \mathrm{E}-03$ \\
\hline $\begin{array}{l}\text { Proteolysis involved in cellular protein catabolic } \\
\text { process }\end{array}$ & 2.76E-03 \\
\hline Cellular protein catabolic process & 2.76E-03 \\
\hline Generation of precursor metabolites and energy & 3.36E-03 \\
\hline $\begin{array}{l}\text { Energy derivation by oxidation of organic } \\
\text { compounds }\end{array}$ & 3.36E-03 \\
\hline Ribonucleoprotein complex biogenesis & 3.65E-03 \\
\hline Vesicle-mediated transport & 5.40E-03 \\
\hline $\begin{array}{l}\text { Regulation of alternative nuclear mRNA splicing, via } \\
\text { spliceosome }\end{array}$ & $7.43 \mathrm{E}-0$ \\
\hline $\begin{array}{l}\text { Transcription initiation from RNA polymerase ॥ } \\
\text { promoter }\end{array}$ & $8.09 \mathrm{E}-0$ \\
\hline Cellular biopolymer catabolic process & \\
\hline
\end{tabular}

Gene Ontology (GO) analysis for stable transcripts (class I in Figure 3) in early embryos using GO::TermFinder. We report significant $\mathrm{GO}$ terms and associated $P$-values unique to stable mRNAs. Cutoff $P$-value $=0.01$. 
Table 3 Regulating the regulators

\begin{tabular}{ll}
\hline Process or pathway & mRNA decay targets in embryos \\
\hline miRNA pathway & Dcr-1 \\
piRNA pathway & $a u b$, piwi \\
RNAi/siRNA pathway & Dcr-2, r2r2, vig, spn-E, armi, Fmr1 \\
Nonsense mediated mRNA decay & Upf1, btz, Smg6 \\
& \\
5' -to-3' mRNA decay & pcm (Xrn 1), Dhh1 \\
3' -to-5' mRNA decay & Rrp4, Rrp42, Rrp45 \\
Deadenylation & twin (ccr4), pop2, Not1 \\
Decapping & Dcp2
\end{tabular}

Transcripts of key proteins in mRNA decay pathways are degraded in early fly embryos. miRNA, microRNA; piRNA, piwi-interacting RNA; RNAi, RNA interference; siRNA, small interfering RNA.

Actively translated mRNAs were enriched in stable mRNA classes (I, stable + transcription) and depleted in decay classes (II to V, decay superclass II-V) (Figure 6a). Conversely, translationally silent mRNAs were enriched in unstable mRNA classes. We concluded that stable mRNAs tend to be translated while mRNAs that suffer degradation are translationally silent; this pointed to a coordinated down-regulation of genes at both the mRNA stability and translation level.

A similar enrichment profile was observed for up- and down-regulated proteins (Figure $6 \mathrm{~b}$ ): genes encoding up-regulated proteins were enriched in stable mRNA classes (I, stable + transcription) and depleted in RNA decay classes (II, III, V; superclass II-V). Conversely, genes of down-regulated proteins were enriched in decay classes (III, V; superclass II-V). Overall, RNA stability was positively correlated with active translation and rising protein levels, while RNA decay was associated with translational silence and protein degradation. These observations suggested a coordination of several post-transcriptional regulatory events to promote the rapid removal of maternally provided gene products (both mRNA and protein) during the first hours of Drosophila development.

\section{Analysis of cis-regulatory motifs mediating RNA degradation}

Our transcript classification system informs us about the degradation behaviors of various sets of transcripts situated in distinct biochemical environments within unfertilized eggs and embryos. Such transcripts are expected to possess particular sequence elements (motifs) that allow them to engage in specific RNA degradation processes or be immune to them. We reasoned that the partitioning of all mRNAs according to maternally and zygotically provided decay activities (Figure 3a) might facilitate the discovery of motifs related to transcript stability and degradation. To test this, we analyzed the 3'
UTRs in different transcript classes using SYLAMER software [70]. Here, lists of mRNAs, ranked by net decay (Figures 2 and 4), were linked with their 3' UTRs as retrieved from the ENSEMBL database. We then analyzed the resulting lists of ranked 3' UTRs for overrepresented motifs of word lengths 6 or 8 . We show $-\log 10$ of the $P$-values for motifs enriched in instable mRNAs as a landscape over 40 cumulative bins (Figure 7a-c).

Comparing across 3' UTRs of all preloaded transcripts, this analysis did indeed detect several motifs associated with severe decay patterns (Figure 7a; see also Figure 3c). By limiting ranked 3' UTR lists to only stable and maternally degraded mRNAs as detected in unfertilized eggs (Figure 7b) or zygotically degraded mRNAs detected in embryos (Figure 7c), we were able to detect further motifs, some of which were associated with exclusively maternal or zygotic degradation. In total, 27 motifs were recovered using the SYLAMER approach. Notably, all (27 of 27) these motifs were complementary to miRNAs identified in Drosophila or other metazoans (Supplementary Table 6 in Additional file 1), suggesting that miRNAs might contribute to the degradation of instable mRNAs. Furthermore, GO analysis of groups of transcripts including decay-associated motifs 1 to 27 showed that almost $50 \%$ of these transcript groups (13 of 27) shared enriched GO terms with unstable mRNAs (Supplementary Figure 9 in Additional file 1, Table 1). Focusing on those transcript groups with higher representation ( $\geq 100$ transcripts), we saw that the proportion of groups sharing GO terms with unstable transcripts rose to $>75 \%$ (13 of 17). These observations strengthened the possibility that the recovered motifs were bona fide cis-regulatory elements associated with RNA instability.

AU-rich elements (AREs) have been shown to elicit mRNA decay in early frog development and Drosophila S2 cells [40] and are positively correlated with mRNA decay in human cells [13]. To explore their role during the first $3 \mathrm{~h}$ of fly development, we linked transcripts with AREs as identified in a recent genome-wide screen [71] to our mRNA decay classes and found that mRNAs with AREs were enriched in decay classes II, IV and V (Figure 7d). This observation suggests that AREs might act as cis-regulators of mRNA turnover in early fly embryos, and would be consistent with a previous study reporting an enrichment of ARE-like motifs in the 3' UTRs of degraded transcripts [46].

\section{Analysis of trans-regulators of RNA decay}

Only a handful of trans-acting factors of mRNA decay turnover are known in Drosophila; these include the mRNA binding proteins (RBPs) Pumilio and Smaug (reviewed in [40]) as well as miRNAs of the miR-309 cluster [72]. 


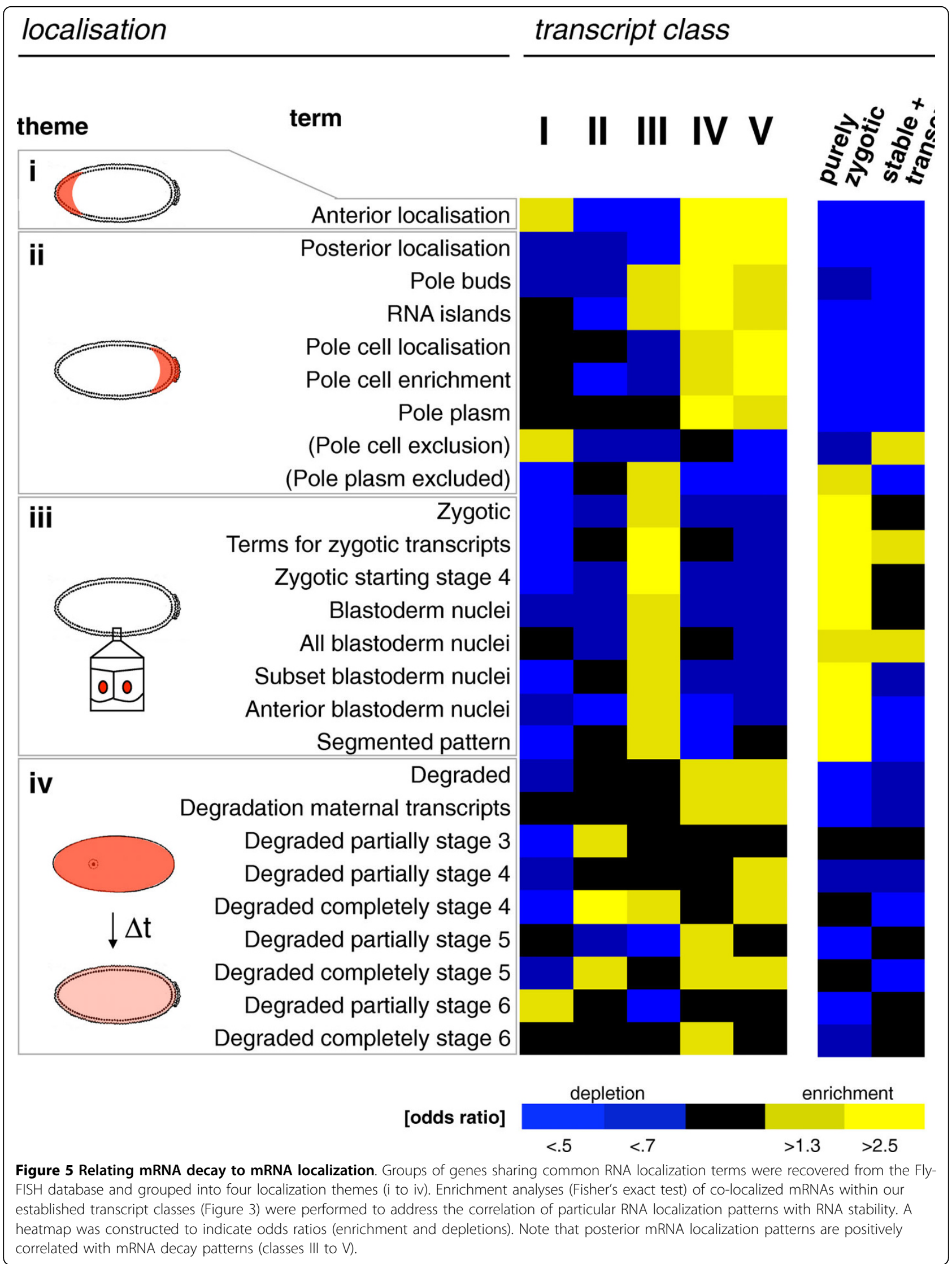


(a)

\section{transcript class}
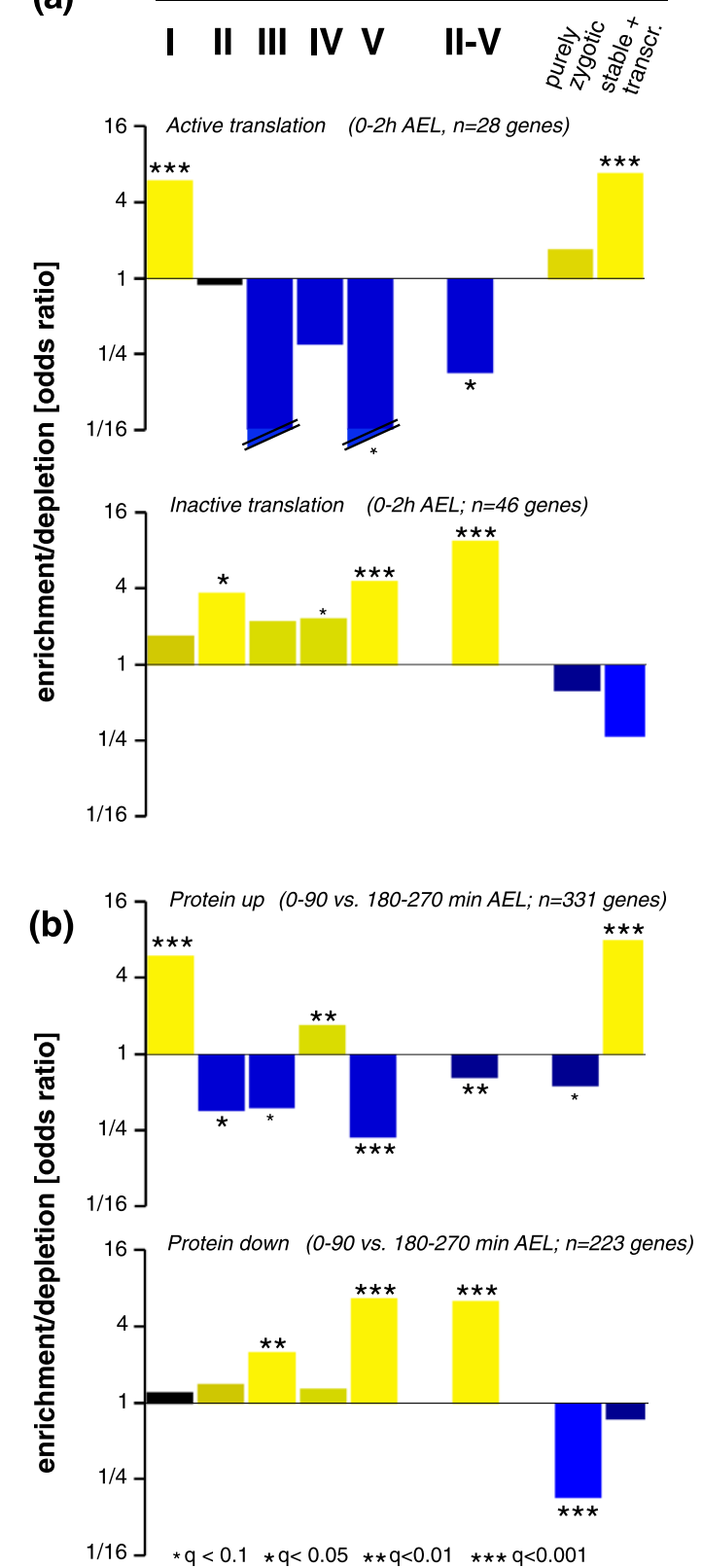

Figure 6 Coordination of RNA and protein turnover. (a) Groups of genes with actively translated or translationally silent mRNAs in early Drosophila embryos were recovered from a genome-wide ribosomal profiling study [68]. Enrichment analyses (Fisher's exact test) were performed to address the correlation between translation rate and RNA stability. Odds ratios (enrichments and depletions) within transcript classes (Figure 3; II-V, union of classes II to $\mathrm{V}$ ) are shown on a $\log 2$ scale (y-axis); color code is as in Figure 5; significance of enrichments are indicated by multiple testing corrected $P$-values (q-values). (b) A recent proteomics screen identified up- and down-regulated proteins in early fly embryos [69]. Enrichment analyses were performed to address the correlation between protein level changes and RNA stability. Note that RNA decay is negatively correlated with active translation and protein up-regulation.
To investigate the ways in which the behavior of mRNAs in our degradation classes relate to the known targets of trans-acting mRNA decay regulators, we performed an enrichment analysis for experimentally validated mRNA target sets of these regulators in our mRNA stability classes (Figure 8a-c). The expression patterns of these regulators as described in the literature are shown in the insets $[28,40,72,73]$.

We found significant enrichment for Pumilio targets only in classes with zygotic decay contributions (classes IV and V; Figure 8a), consistent with its proposed role in zygotic decay activities [46]. The minor enrichment in Pumilio targets within the stable mRNA class (class I) was statistically insignificant.

Smaug targets were significantly enriched in classes III and $\mathrm{V}$, both of which show maternal decay activities, while no enrichment is seen in the exclusively zygotic decay class IV (Figure 8b). These observations are in line with the maternal origin of Smaug [28]. Given that Smaug had been shown to be an important, maternally provided mRNA decay factor [43,74], the absence of significant $(P=0.42)$ enrichment of its targets within the 'maternal decay only' class (II) was somewhat unexpected. The maximum enrichment (13-fold) of Smaug targets was detected in the 'mixed decay' class (V). A plausible explanation for these observations might be that Smaug requires additional, zygotic decay factors to perform its normal functions; alternatively, Smaug targets might be targeted by zygotic factors in a Smaugindependent fashion.

We found enrichments for miR-309 cluster targets only in classes with zygotic decay contributions (classes IV and V), consistent with the strictly zygotic provision of miR-309 cluster miRNAs [28]; the strongest enrichment was observed in the mixed decay class $(\mathrm{V})$. This latter finding suggested to us that miR-309 cluster targets might be targeted by additional, maternally provided mRNA decay factors. To investigate this further, we separately computed maternal and zygotic decay contributions for approximately 400 miR-309 cluster targets [72] and show them for all genes as a scatterplot (Figure 8d). This demonstrated that most of the miR-309 cluster targets show, indeed, maternal decay contributions. This points to a common interaction of miRNAs of zygotic origin with preloaded, maternal mRNA decay factors.

We note that there was generally no significant enrichment of any decay targets in the stable mRNAs (class I) or transcribed, non-degraded mRNAs (purely zygotic, stable + transcription). In contrast, we often observed highly significant depletion for decay targets in these classes (Figure 8a-c).

Taken together, the specific target enrichment patterns for experimentally validated RNA decay regulators 

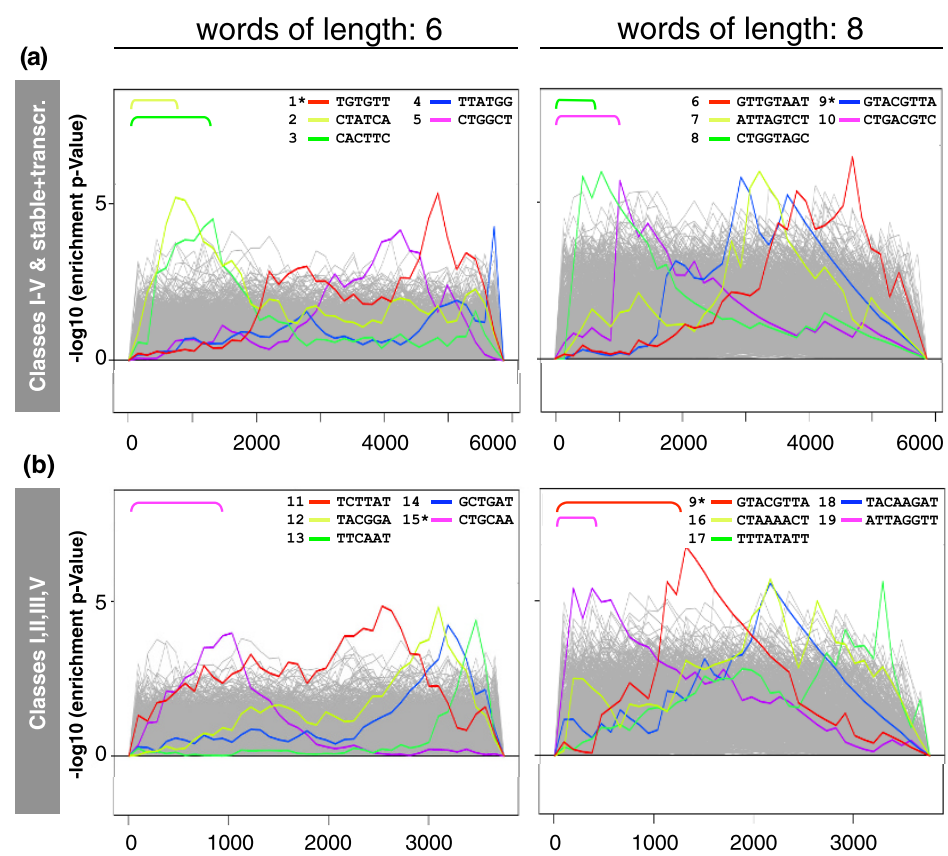

(c)
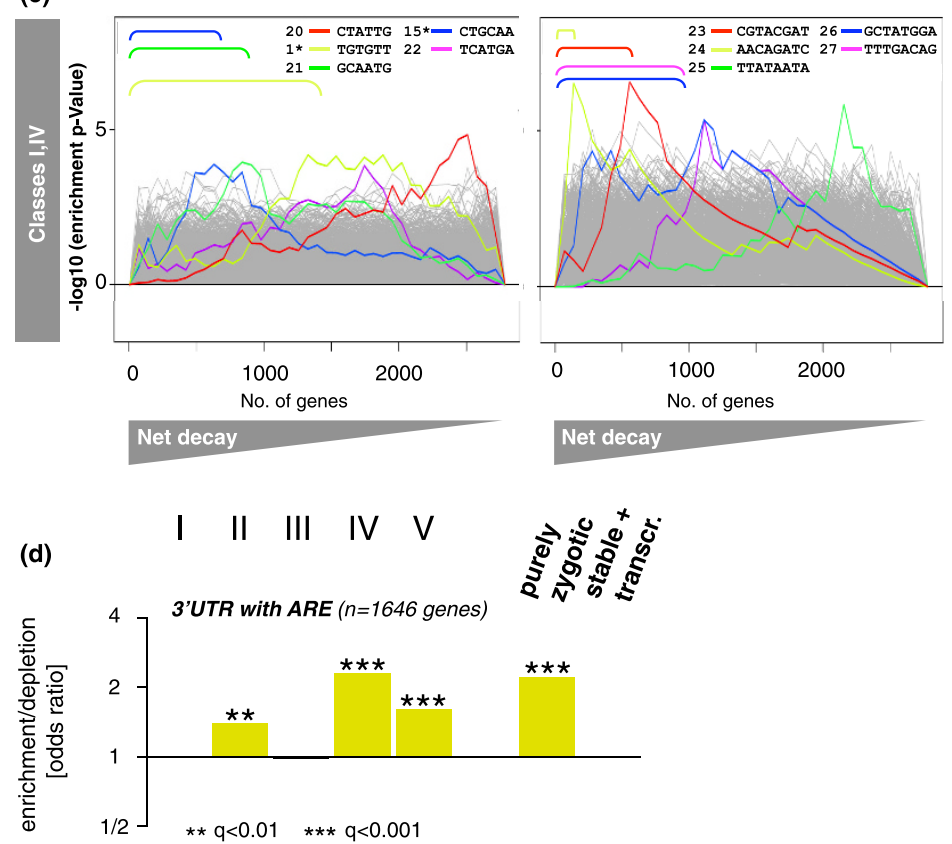

Figure 7 Cis-regulators of mRNA decay in early Drosophila embryos. (a-c) Motif discovery in 3' UTRs using SYLAMER [70]. Genes were ranked by mRNA net decay values (Figure 2) and enrichment analyses for words of lengths 6 and 8 were performed; - $\log 10$ of enrichment $P$ values (y-axis) are plotted for words enriched in 3' UTRs of unstable mRNAs (x-axis). P-value profiles for the top five enriched motifs are highlighted and shown for each enrichment analysis; a total of 27 unique motifs is shown (asterisk indicates motifs recovered in more than one enrichment). For a peak occurring on the positive y-axis, the corresponding word is overrepresented in the $3^{\prime}$ UTRs for the genes to the left of that peak (colored brackets) while the word is underrepresented in the genes to the right. Note that all motifs (1 to 27) are complementary to seed sequences of characterized miRNAs (Supplementary Table 6 in Additional file 1). Enrichment analyses are shown for: (a) all transcripts preloaded onto the oocyte (Figure 3); (b) stable and maternally degraded mRNAs; and (c) stable and zygotically degraded mRNAs (compare Figure 3). (d) mRNAs with AU-rich elements (ARE) were recovered from a genome-wide screen [71]. An enrichment analysis (Fisher's exact test) was performed to address the correlation between AREs and RNA stability. We found that RNA decay (classes II, IV and V) is positively correlated with the presence of AREs in transcript 3' UTRs. Odds ratios (enrichments and depletions) within transcript classes (Figure 3) are shown on a log2 scale (y-axis); color code as in Figure 5; significance of enrichments is indicated by multiple testing corrected $P$-values (q-values). 


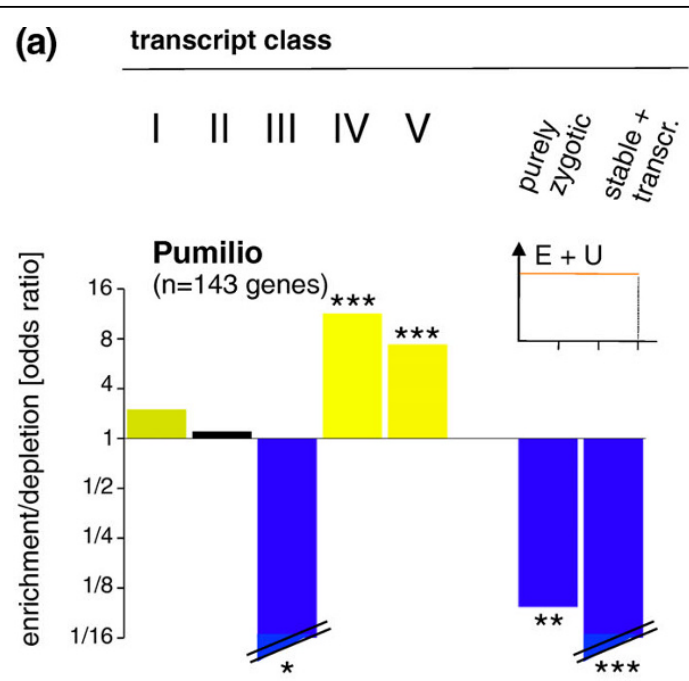

(b)

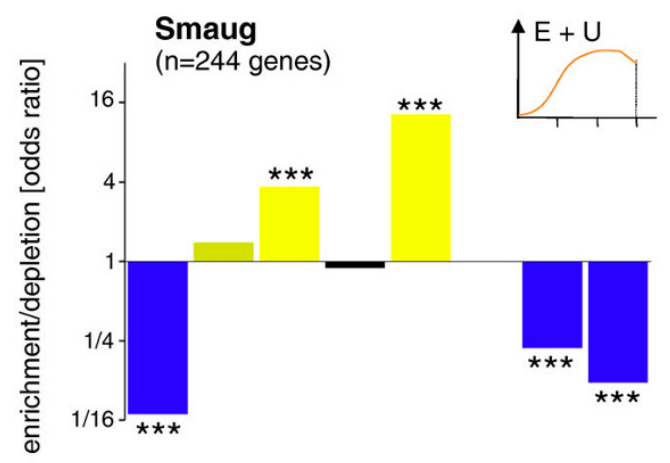

(c)

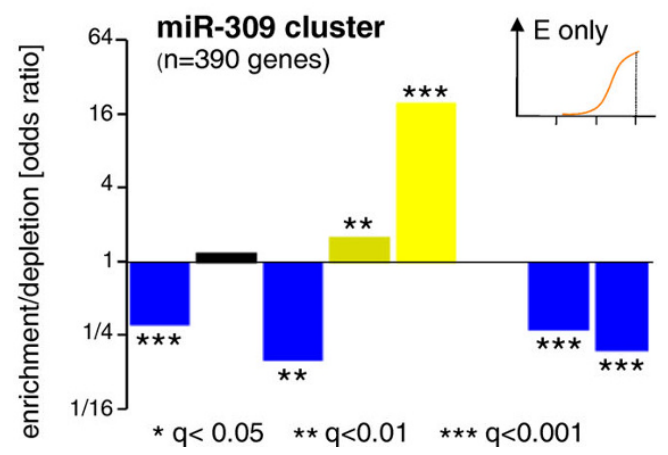

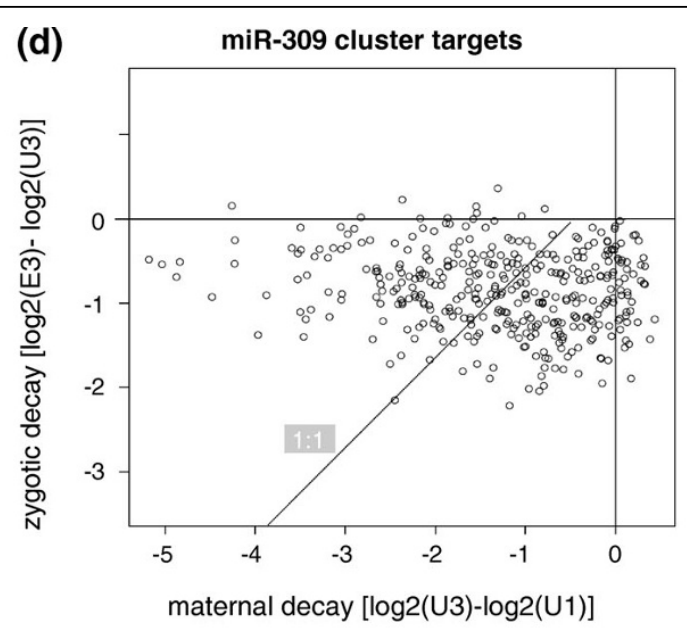

Embryonic expression

(e) (modified after Ruby et al, 2007) enrichment

Target
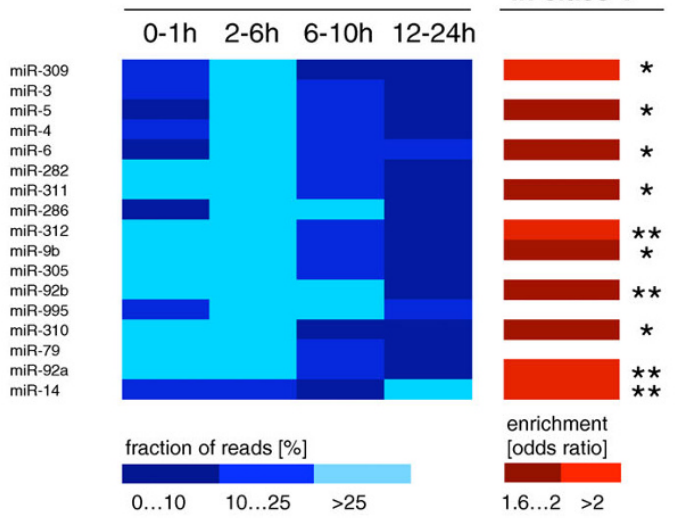

(f)

mRNA half-life [min] (2-3h AEL)

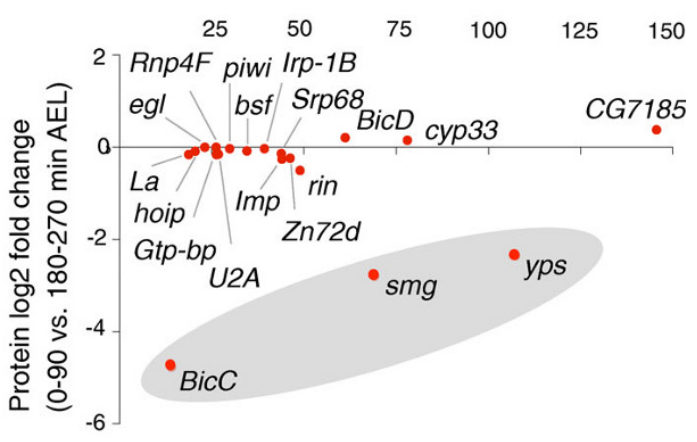

Figure 8 The relationship between mRNA decay, mRNA binding proteins and miRNAs. (a-c) Enrichment analyses (Fisher's exact test) were performed for genome-wide mRNA target sets of Pumilio [109], Smaug [43] and miR-309 cluster miRNAs [72] within our transcript classes (Figure 3). Expression dynamics of the regulators during the first $3 \mathrm{~h}$ AEL are indicated (see insets; units on $x$-axis are hours AEL). Odds ratios (enrichments and depletions) are shown on a log2 scale (y-axis); color code as in Figure 5; significance of enrichments is indicated by multiple testing corrected P-values (q-values). (d) miR-309 cluster targets: maternal decay plotted against zygotic decay. Note that most of the mRNA targets show maternal decay contributions. The dotted line represents the 1:1 ratio of maternal and zygotic decay. (e) miRNAs with strong expression restricted to early embryos; odds ratios of miRNA target set enrichment within the mixed decay class $(V)$ and significance levels ( $q$ values) are indicated. Embryonic expression modified after Ruby et al. [75]. (f) mRNA binding proteins (RBP) with dynamic expression (short mRNA half-life, protein log2 fold-change) in early embryos (see text for details). Grey shading highlights RBPs with both low mRNA half-lives and drops in protein levels. 
in the stability classes established in this study (Figure 3) are in good agreement with their well-known expression dynamics; these findings supported the biological relevance of our classification.

Given the great diversity of mRNA decay patterns detected in this study (Figure 2), the lack of significant overlap of target sets for Pumilio, Smaug and miR-309, and the fact that these regulators do not seem to target thousands of unstable transcripts (data not shown) led us to predict that other, yet uncharacterized mRNA regulators must be active during early Drosophila embryogenesis. To advance these considerations, we searched for candidate miRNA and protein regulators whose expression is consistent with a role in mRNA turnover during the first hours of fly development (Figure 8e, f). An essential condition for a functional mRNA decay regulator is that it temporally co-exists with its targets, and that upon action on them, these are reduced in their expression level. After functional contact with targets occurs, expression of the regulator is unrestricted and may diminish or vanish altogether. For example, previous work reported that miRNAs from the miR-309 cluster are synthesized anew in early Drosophila embryos (Figure 8c), triggering the decay of hundreds of mRNAs [72]; genetic removal of the miR-309 cluster leads to a stabilization of these targets. To identify other miRNAs with a potential role in mRNA decay control, we turned to recently published miRNA RNA-Seq data collected from a Drosophila developmental series including early, mid- and late embryogenesis; here, the authors isolated total RNA including small RNA collections and applied a next-generation-sequencing approach to identify and quantify miRNAs expressed throughout development [75].

As miR-309 cluster miRNAs are expressed only in early embryos, we filtered the published dataset for miRNAs with similar expression dynamics. Here, we selected miRNAs with significant expression in early embryos ( $>100$ sequencing reads at 0 to $6 \mathrm{~h}$ ) and a decrease in expression at mid- to late embryonic stages and found 17 miRNAs that pass these criteria (Figure 8e). To explore the possible effects that these miRNAs might have on mRNA decay processes in fly embryos, we recovered full lists of predicted targets of these 17 miRNAs from miRBase [76,77] and performed an enrichment analysis for these targets in our decay classes. We found that target sets for the majority of our short-listed miRNAs (10 out of 17) show significant enrichment in our mixed decay class V (Figure 8e); this includes predicted targets for $m i R-6, m i R-5$ and $m i R$ 309 , all of which belong to the miR-309 cluster, and is in agreement with the enrichment patterns of their experimentally validated targets (Figure 8c). Notably, for decay classes other than $\mathrm{V}$, we did not detect any significant enrichments. Two scenarios could explain the exclusive miRNA target set enrichments in the mixed decay class. One possibility is that factors of maternal origin must be complemented by freshly transcribed miRNAs to elicit effective mRNA degradation; alternatively, miRNAs themselves might represent the maternal component that would require the zygotic production of additional decay factors. Excluding the miR-309 cluster miRNAs, for which zygotic transcription has been demonstrated as the only source [72], we are at present unable to distinguish which one of these possibilities should be the most likely. Recent experiments in the mouse demonstrating the suppression of miRNAs in mature oocytes $[78,79]$ would suggest that - should both systems be comparable - the miRNA component is only active after the onset of zygotic transcriptions.

We also looked at candidate protein regulators seeking to identify RBPs whose expression is consistent with a role during mRNA turnover in Drosophila embryos. A recent survey of the literature found that many yet uncharacterized RBPs are expressed during fly embryogenesis [80]; studies in yeast suggest that RBPs targeting large groups of mRNAs show generally high protein abundances [81].

Here, we had to confront the fact that beyond their need for mRNA binding properties, not much is known about the common features of protein regulators of mRNA decay. Nevertheless, one salient attribute of the few proteins with proven roles in mRNA degradation appears to be the dynamic nature of their expression patterns [81]. An example of this in flies is Smaug, a major contributor to maternal mRNA decay activities $[28,43]$. Although the ultimate explanation of how a highly dynamic expression pattern relates to the molecular function of an mRNA regulator is still missing, we used this correlation do develop an approach to identify those RBPs with a potential role in mRNA turnover. For this, we first recovered a list of all annotated and predicted mRNA binding proteins (GO:0003729) from Flybase [82] (December 2009) and linked these to their respective mRNA half-lives and protein turnover rates (transcript half-lives were calculated in this study (Figure 4) and protein $\log 2$ fold-changes between 0 and $90 \mathrm{~min}$ utes AEL and 180 and270 minutes AEL were obtained from a recent proteomics screen in Drosophila embryos [69]; Supplemental Figure 8 in Additional file 1). We retained genes for which mRNA and protein data were available, that had mRNA half-lives below 150 minutes, and for which at least five quantified peptides were reported in the proteomics study. We then plotted mRNA half-lives against protein log2 fold-changes for this subset of genes (Figure 8f).

This analysis recovered smaug (smg) as one of the most dynamically expressed genes at both the protein and mRNA level and identified approximately 20 
additional RBP encoding genes with dynamic expression at the RNA level in early embryos. Two of these $(B i c C$, yps) showed in addition a significant drop in protein levels similar to smg. It will be important to establish whether these well-characterized post-transcriptional regulators, which have previously been implicated with translational repression, RNA localization and, in the case of yps, splicing [83-89], play an additional role in RNA stability control.

Overall, we identified candidate miRNAs and RBPs whose expression is consistent with a role in mRNA degradation (Figure 8e, $\mathrm{f}$ ) and present evidence that ten miRNAs expressed in early embryos negatively affect mRNA levels during the first hours of development (Figure 8e). The strong and often exclusive enrichments for experimentally validated or predicted targets of both RBPs (Figure $8 \mathrm{~b}$ ) and miRNAs (Figure 8c, e) within the mixed decay class $(\mathrm{V})$ are consistent with the notion that mRNAs are commonly targeted by more than one decay regulator of maternal or zygotic origin.

\section{Experimental validation of cis- and trans-mRNA decay regulators}

To experimentally validate our bioinformatic analyses, we focused on two sets of experiments aimed at establishing the roles of cis-and trans-regulators of RNA degradation in an in vivo system.

To investigate cis-regulation we selected the gene cortex (cort), whose mRNA is one of the most severely degraded and most short-lived species detected in our analysis (Figure 4d). To determine the primary sequences contributing to the adoption of the cort RNA pattern, we developed an in vivo assay that combines microinjection of supercoiled plasmid DNA luciferase reporter constructs into Drosophila embryos with luminometric quantification of reporter expression determined in protein extracts derived from single Drosophila embryos (Figure 9a). We used this system to test the performance of two firefly-luciferase (F-luc) constructs: one in which coding sequences for F-luc were coupled to the 3' UTR of $\alpha$-tubulin $84 B(\alpha-t u b)$, a very stable mRNA according to our study; and another construct in which F-luc coding sequences were linked to the 3' UTR of cort, a very unstable mRNA in our study (Figure 9b, c). Both these constructs were driven by a sisA promoter that supports expression in early embryos (Figure 9b) [90]. Enzymatic activity derived from these two constructs was compared to that of a Fluc control carrying only an SV40 3' UTR sequence (Figure 9c). To control for embryo-to-embryo variation affecting total injected volumes of plasmid solutions, we compared the performance of the F-luc constructs described above with a co-injected reference construct encoding Renilla-luciferase (R-Luc) (Figure 9c).
These experiments revealed that the presence of $\alpha$-tub 3' UTR sequences did not affect the median reporter activity compared to the control constructs (Figure 9d). In contrast, cort 3' UTR sequences significantly decreased reporter activity (Figure 9d). We therefore concluded that the transfer of cort 3' UTR sequences to a heterologous reporter system is able to mimic the expression dynamics of cort transcripts detected in our genome-wide analysis (Figure 9b).

Our study suggested that several miRNAs might be involved in the control of RNA degradation during early Drosophila development (Figure 8e). To establish whether modulations of miRNA level had an impact on RNA degradation patterns in vivo, we focused on miR14 , which is known to be present during early embryogenesis [75] and has multiple predicted targets within our instable RNA classes. If during normal development a particular miRNA promotes the degradation of its target mRNAs, we inferred that genetic removal of such miRNA from the system would lead to the stabilization of its mRNA targets. To test this hypothesis, we studied the expression of $\mathrm{Hr} 78$, an unstable mRNA (Figure 10a) predicted to be targeted by miR-14 (Figure 10b) in embryos with two (wild type), one (heterozygous mutant) and no genomic copies (homozygous mutant) of miR-14 (Figure 10c). Analysis of the expression levels of $\mathrm{Hr} 78$ by semi-quantitative RT-PCR revealed that, indeed, $H r 78$ mRNA stabilization depends on miR-14 dosage, with highest expression in the homozygous mutant background, intermediate expression in the heterozygous condition and lowest expression levels in the wild type (Figure 10c). These results are consistent with an active role of miR-14 in RNA stability control during early Drosophila development, as predicted by our study.

\section{Discussion}

This study investigates how mRNA degradation is controlled during Drosophila early development. Our experimental design, involving the sampling of mRNAs from embryos and unfertilized eggs, allowed us to determine the degradation patterns of all transcripts present during early fruit fly development, and to tease apart the contributions of maternally and zygotically encoded factors to the process of mRNA decay. Our results provide kinetic parameters for the degradation of thousands of mRNAs, and establish the ways in which mRNA decay relates to mRNA localization, protein turnover and gene function in Drosophila. We also detected enrichments for cis-regulatory sequences in transcripts showing common degradation patterns and propose specific proteins and miRNAs as developmental regulators of mRNA decay during early embryogenesis. We also validate the roles of some of these regulators and 3' UTR regions 


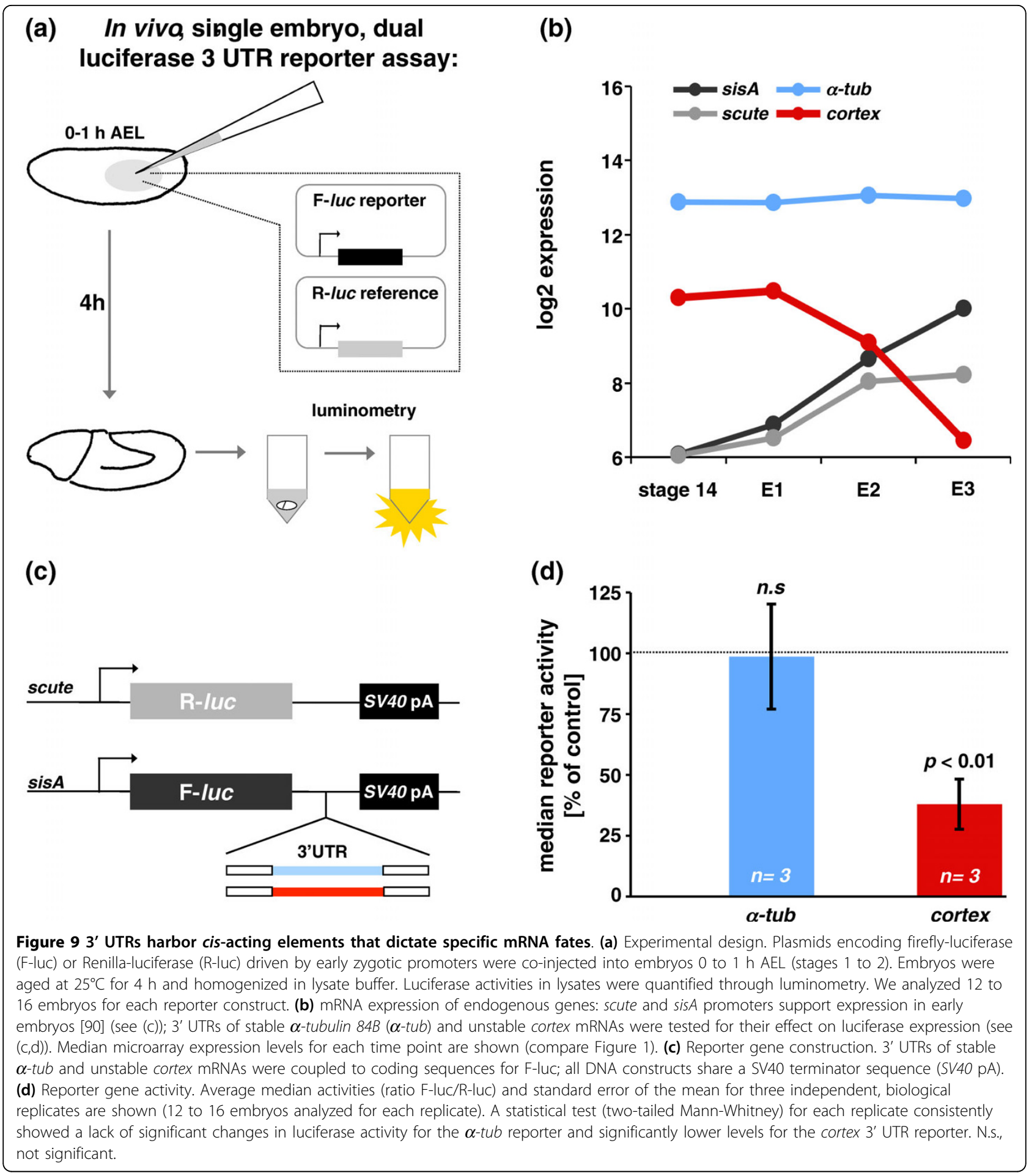

experimentally. Here, we discuss how our work relates to previous studies investigating mRNA degradation in cell culture systems and to what is known about mRNA degradation control in Drosophila, and the ways in which our study contributes to the understanding of the molecular mechanisms of mRNA decay.

\section{mRNA degradation in cell culture systems}

Previous work in cell culture revealed important features of the process of prokaryotic and eukaryotic mRNA decay. Genome-wide studies in bacterial cultures $[60,64]$, yeast $[12,16]$, Drosophila Schneider cells $[11,91]$ and various human cell lines $[13,57,62]$ showed that (in 


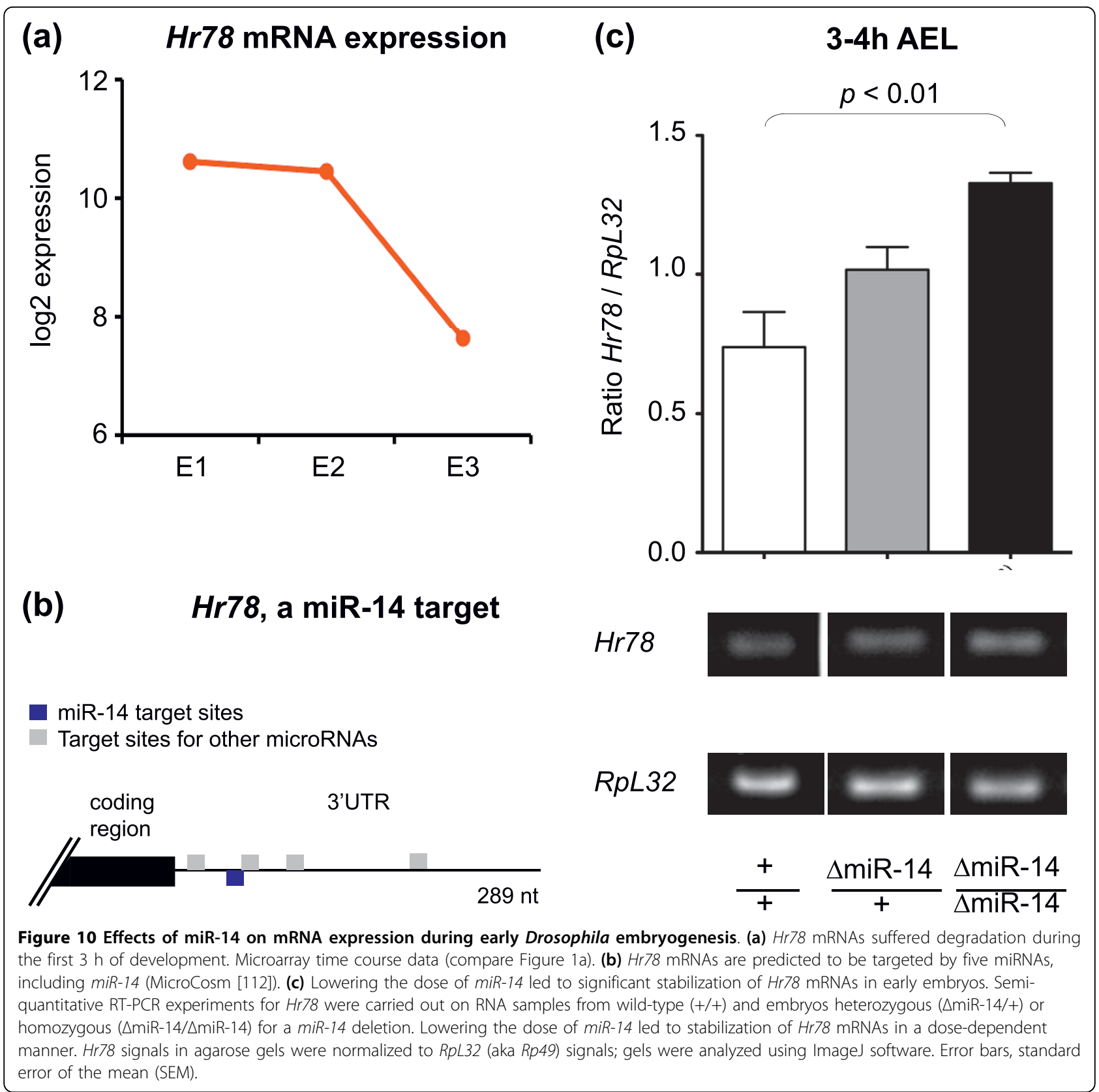

every system) mRNA half-lives are very diverse. For instance, half-lives from circa 3 minutes to $>100 \mathrm{~min}$ utes were observed in yeast [12] while in human cells they ranged from $<30$ minutes to $>24 \mathrm{~h}$ [13]. This level of diversity in mRNA degradation rates would be consistent with significant and dynamic regulation of mRNA decay across a wide range of organisms.

These experiments in cultured cells also revealed that unstable transcripts tend to encode products with specific cellular functions or processes, as similar functional themes appear related to unstable transcripts in organisms as diverse as yeast and humans. For example,
mRNAs encoding products related to cell cycle control, transcription and mRNA processing are generally unstable while genes encoding factors involved in protein synthesis produce stable mRNAs in human and yeast $[13,58]$. The similarities observed across these studies could, in principle, be the result of evolutionary conservation in the ways mRNA degradation relates to gene function. Alternatively, they may reflect common adaptations developed by each system to the conditions of cell culture. Our results showing that unstable mRNAs are associated with the replication machinery and enriched for cell cycle regulators in fly embryos 
(Table 1) provide support to the first interpretation. The lack of departure from ancient associations between mRNA decay and gene function, in turn, implies an important role of mRNA degradation in cellular function, as genes encoding products with particular functions appear forced to retain common mRNA decay patterns.

We hypothesize that, in the Drosophila embryo, the low stability of mRNAs encoding cell cycle and DNA replication factors might be linked to the timely elimination of cell cycle regulators known to be crucial for the slowing down of mitotic cycles at the onset of gastrulation [27,28] (Figure 1a) and reflect a need to readjust their expression levels once cell divisions become restricted to particular subdomains of the embryo $[92,93]$.

\section{mRNA degradation during Drosophila development}

Microarray experiments in Drosophila embryos established that major developmental transitions (for example, gastrulation, end of the dorsal closure, imaginal disc formation) are mirrored by global changes in gene expression. In particular, these studies showed that down-regulation of maternally provided mRNAs occurs in two distinct temporal waves taking place at early and mid-embryonic stages [50,51]. Further genomewide studies in Drosophila provided the first hints on the causes underlying the temporal control of maternal mRNA decay. Tadros et al. [43] detected some of the targets of the maternal RNA decay machinery in unfertilized Drosophila eggs and, notably, identified the RBP Smaug as an important factor controlling maternal mRNA decay; unfortunately, the wide temporal windows used for sampling in this study $(2 \mathrm{~h})$ lacked the needed resolution for the analysis of dynamic early mRNA decay events (Figures 2, 3 and 4). De Renzis et al. [46] employed compound chromosomes to produce embryos lacking single chromosomes (or chromosome arms) to look at the effects of these deficiencies on the transcriptome at a single time point (mitotic cycle 14; Supplementary Figure 8 in Additional file 1), allowing the identification of maternal and zygotic contributions to total mRNA levels at this particular stage. However, this study provided no information on how these results relate to earlier or later events. Although these studies significantly advanced our understanding of the mRNA degradation processes taking place during early Drosophila development, they did not determine the separate contributions of maternal and zygotic decay factors to mRNA turnover during early development. The first study focused on unfertilized eggs and maternal decay activities only [43]; in contrast, the experimental design used in our present study (sampling both embryos and unfertilized eggs) allowed us to capture the full complement of maternally and zygotically controlled RNA decay patterns at high temporal resolution and to tease apart the contributions of maternal and zygotic factors to mRNA turnover during early development.

Our analyses revealed that most $(60 \%)$ of the preloaded mRNAs suffer rapid degradation during the first $3 \mathrm{~h}$ of development (Figure $3 \mathrm{~b}$ ), frequently leading to reductions below $50 \%$ of initial mRNA levels (Figure 2 and 4). Notably, we show that more than 1,500 mRNAs are targeted by both maternal and zygotic decay factors (one-third of all decay events; Figure 3b, class V); consistent with additive effects of this dual action and in line with previous observations on specific genes [22], mixed decay patterns are more severe than exclusively maternal or zygotic decay patterns (Figure $4 \mathrm{~b}, \mathrm{c}$ ). In addition, we found that mRNA decay during the first 3 $\mathrm{h}$ is largely dominated by maternal decay activities as mRNA classes with maternal decay contributions show the most severe decay patterns (Figure $4 \mathrm{~b}, \mathrm{c}$ ).

We also showed a linkage between mRNA stability control and mRNA localization (Figure 5). Notably, 125 out of 198 (63\%) transcripts with annotated posterior localization in the Fly-FISH database show detectable levels of degradation during the first $3 \mathrm{~h} \mathrm{AEL}$, suggesting that mRNA decay might contribute to their localized expression in embryos. These results are coherent with previous observations on specific mRNAs that are localized to the posterior of the embryo by a 'degradation/ protection' mechanism [22,39].

Secondly, we detected approximately 400 mRNAs with particularly strong degradation and concomitant transcription (Figures 3b (class III) and $4 \mathrm{~b}, \mathrm{c}$ ), supporting the idea that the removal of ubiquitously distributed maternal mRNAs combined with localized, albeit limited (Supplementary Figure 5 in Additional file 1), zygotic transcription is an efficient way to create localized patterns of gene activity in the early embryo [46].

Our analyses also revealed a coordination of protein and RNA turnover in early embryos as genes encoding translationally silent mRNAs and proteins with decreasing levels in early embryos are enriched in unstable transcript classes (Figure 6). This points to a concerted effort at several post-transcriptional levels to rapidly remove a subset of maternally provided gene products from the developing embryo during early development.

\section{Mechanisms of mRNA turnover in Drosophila embryos}

In mechanistic terms, the control of mRNA degradation requires the binding of trans-regulators to cis-regulatory motifs in target mRNA sequences; until now, very few cis- and trans-regulators of mRNA turnover have been identified in Drosophila embryos (reviewed in [40]); this is also true for most other systems. 
The difficulties in identifying and predicting novel cisregulatory motifs of mRNA decay could be due to several reasons. They could be a manifestation of the lack of a simple molecular code relating the mRNA decay regulators with primary sequence elements in their targets: perhaps efficient mRNA decay regulation requires a specific combination of primary sequence and mRNA secondary structure adopted by mRNA targets. Alternatively, a complex mixture of RBPs and miRNAs might be needed to determine a specific mRNA decay output: as many regulators are needed, sequences and structures in target mRNAs must conform with various overlaying sets of rules that are not easy to unveil. In all cases, refinement of software packages able to scan for both primary and secondary structure motifs, perhaps linked to simultaneous co-variance analysis associated with stem regions within hairpin-loop structures, might be able to decode the cis-elements responsible for mRNA decay. A condition for the development of these computerized approaches is the availability of large datasets grouping transcripts with common mRNA decay patterns. Our work here modestly contributes to this, providing high-resolution mRNA decay profiles for approximately 4,000 genes (Figure 3) obtained in the physiological context of Drosophila development.

Regarding cis-regulators of mRNA decay, we demonstrated that unstable mRNA classes are enriched for transcripts with AREs (Figure 7b); this is consistent with a conserved role for AREs in mRNA decay control during early embryogenesis in both flies [46] and frogs [94]. We also detected motifs enriched in 3' UTRs of unstable mRNAs (Figure 7a-c), many of which are complementary to miRNA seed regions (Supplementary Table 6 in Additional file 1). For selected genes we demonstrated that 3' UTR sequences are sufficient to recapitulate the fate of the endogenous mRNA when coupled to a heterologous reporter in early embryos (Figure 9), suggesting that crucial cisregulatory elements for RNA stability control are likely to reside in this part of the transcripts.

Previous work had identified a few trans-regulators of mRNA decay in early embryos, including the RBPs Pumilio and Smaug and miRNAs of the miR-309 cluster (Figure 8a-c) [43,72,73,95]. Mining previously published miRNA deep sequencing data [75] and linking RNA decay parameters (this study) with proteomics data for individual genes, we identified several other miRNAs and RBPs whose expression is consistent with a role in mRNA decay control (Figure 8e, f) and present evidence linking candidate miRNAs with mRNA decay patterns in early embryos (Figure 8e). We confirmed the activity of one predicted RNA decay regulator, miR-14, in early embryos experimentally (Figure 10).
Importantly, we show that targets of both RBPs (Figure $8 \mathrm{~b}$ ) and miRNAs (Figure 8c, e) are enriched in mixed decay classes, and that the majority of transcripts targeted by zygotic miRNAs derived from the miR-309 cluster were concomitantly targeted by maternal decay factors (Figure 8d). In addition to this, we found that more than 1,500 mRNAs require a combination of degradation factors encoded by the mother and the zygote (Figure $3 \mathrm{~b}$ ). Altogether, these results support the hypothesis of a complex mixture of RBPs and miRNAs determining particular mRNA decay outputs.

Alternatively, the maternal machinery may provide a ground-state decay mechanism with few specificity factors, and zygotic components could provide specificity molecules that lead to recognition of certain subsets of mRNAs, enhancing their association with the maternally provided decay machinery. This model has the attribute of requiring just a single regulatory molecule for the degradation of a message in a manner dependent on maternal or zygotic factors. A third explanation could be that mRNAs degraded by both zygotic and maternal factors interact with generic mRNA destabilizing factors, which enhance both maternal and zygotic decay pathways, rather than specific ones that preferentially use one pathway over the other.

In sum, our work advances the current understanding of the processes controlling mRNA degradation during early Drosophila development, taking us one step closer to the understanding of mRNA decay processes in all animals. Our data should also provide a fruitful ground for further experimental and computational studies investigating the process of mRNA decay.

\section{Conclusions}

Spatio-temporal modulations in mRNA levels are central for animal development. These modulations in transcript concentration come as a result of two opposing processes: mRNA synthesis and degradation. Our work here combined developmentally timed collections of Drosophila embryos and unfertilized eggs with genome-wide microarray technology to determine the degradation patterns of all transcripts present during early development. Our experiments revealed the kinetics of mRNA decay at early development, the contributions of maternally and zygotically encoded factors to mRNA degradation, and the ways mRNA decay profiles relate to gene functions, mRNA localization patterns, translation rates, and protein turnover. Our transcript catalogues also allowed us to detect cisregulatory sequences enriched in transcripts with common degradation patterns, as well as to propose several proteins and miRNAs as developmental regulators of mRNA decay during early fly development. 
Finally, we validated experimentally the effects of a subset of cis-regulatory sequences and trans-regulators in vivo. In sum, our work advances the current understanding of the processes controlling mRNA degradation during early Drosophila development, taking us one step closer to the understanding of mRNA decay processes in all animals. Our data should also provide a valuable resource for further experimental and computational studies investigating the process of mRNA decay.

\section{Materials and methods}

\section{Fly stocks and culture}

Wild-type embryos were recovered from Oregon Red (OR) flies. Sterile males were recovered from tud[1] bw [1] sp[1] virgins crossed to OR males (Sons of tudor, SOT) $[96,97]$. To collect unfertilized eggs, wild-type OR virgins were mated to SOT males. Hence, both embryos and unfertilized eggs were of identical, wild-type genotype (OR). miR-14 heterozygous and homozygous embryos were recovered from miR-14 $\Delta[1] / \mathrm{CyO}$ [98] (a gift from Stephen Cohen).

\section{Confocal imaging}

Embryos were stained with 4',6-diamidino-2-phenylindole (DAPI) and fluorescein isothiocyanate (FITC)-phalloidin following standard procedures and imaged on a Leica TCS SP5.

\section{RNA sample collections}

Embryos or unfertilized eggs were collected and aged at $25^{\circ} \mathrm{C}$ using standard methods. Mature oocytes were isolated from wild-type fly cultures by a combined blender/ sieving method that allows specific and efficient enrichment of stage 14 oocytes [45,99-101]. Oocyte staging was verified according to King (1970) [102]; the non-activated state was controlled by bleach treatment $[44,45]$ on aliquots of egg collections. RNA was purified using Qiagen (Crawley, United Kingdom) RNeasy Mini Kit.

\section{Microarray hybridization and data analysis Microarray hybridizations}

Affymetrix Drosophila GeneChip 2.0 microarray hybridizations were carried out at the UK Drosophila Affymetrix Array facility at the Sir Henry Wellcome Functional Genomics Facility of the University of Glasgow, UK. Excess RNA was kept for quantitative PCR validation experiments.

\section{Data preprocessing, quality assessments, profile classification and enrichment analyses}

Data preprocessing, quality assessments, profile classification and enrichment analyses were carried out using $R$ [103] and Bioconductor [104]. In brief, raw data were pre-processed applying variance stabilization and normalization (vsn) $[105,106]$, followed by a LOESS regression and probe set summary using robust multichip average (RMA) [107]. Microarray data quality assessments confirmed high data quality (Supplementary materials and methods and Supplementary Figure 1 in Additional file 1). For Figure 1b, a hierarchical clustering (complete linkage, Pearson's correlation) was performed with RMA pre-processed data for all probe sets.

\section{Classification of probe set profiles}

Classification of probe set profiles and the collapsing into classifications for unique genes is described in detail in the Supplementary materials and methods in Additional file 1.

\section{mRNA half-lives}

Decay constant $k$ and half-lives $t_{1 / 2}$ (Figure $4 \mathrm{~b}, \mathrm{~d}$ ) were computed assuming exponential decay between $t_{2}$ and $t_{3}$ of the respective time series (Figure 4a) as $k=-\ln$ $\left(\left(\right.\right.$ Expression $\left.t_{3}\right) /\left(\right.$ Expression $\left.\left.t_{2}\right)\right) / \Delta t_{2,3}$, with $\Delta t_{2,3}=60$ minutes (Figure 1a), and $t_{1 / 2}=\ln (1 / 2) /-k[1]$.

\section{Gene Ontology analyses}

The top 1,000 decay targets with lowest half-lives were identified (Figure 4, $t_{1 / 2}<30$ minutes); 1,677 stable class I (Figure 3) probe sets were collapsed into 1,616 unique genes. The complete set of Flybase annotated genes ( $\mathrm{n}=$ $16,085)$ was considered as background universe. GO analyses were performed using GO::TermFinder [108]. A Bonferroni-correction for multiple testing was applied to enrichment $P$-values.

\section{Enrichment analyses}

An mRNA localization annotation matrix for $>3,000$ genes was recovered from the Fly-FISH website (May 2008) $[66,67]$. We collapsed Fly-FISH annotations for developmental stages covering and slightly exceeding our time series time frame (approximately 0 to $3.5 \mathrm{~h}$ AEL, developmental stages 1 to 7; Supplementary Figure 8 in Additional file 1), yielding groups of genes annotated for 111 different localization terms. We report enrichments for 26 localization terms. Target sets for Pumilio [109], miR-309 cluster miRNAs [28], lists of mRNAs with active or no translation [68], lists of upand down-regulated proteins [69] and a list of genes with AREs [71] were obtained from the literature; for Smaug, we reanalyzed available raw data from the Gene Expression Omnibus [GEO:GSE8910] as described [43] and considered 260 genes with the highest differential expression as targets. For all lists, we retained only genes represented on Drosophila Genome 2.0 Gene Chips. Enrichment analyses were performed using Fisher's exact test; multiple testing was controlled for at a false discovery rate of $10 \%$ [110].

\section{Quantitative and semi-quantitative RT-PCR}

For each experimental condition, a minimum of three technical replicates were performed on at least two 
biological replicate samples. For quantitative RT-PCR, we used SYBR Green I detection format on a Roche Lightcycler 480 platform. Primer sequences are listed in Supplementary Table 1 in Additional file 1. Transcript levels were determined as expression ratios using stable transcripts as reference (Rpl32, Rpl21).

\section{Dual-luciferase assays}

Reporter assays were performed essentially as described [90] (see Additional file 1 for details). 3' UTR sequences were amplified by PCR from cDNA and inserted into a F-luc reporter construct; firefly reporter constructs were co-injected into 0 to $1 \mathrm{~h}$ embryos with a reference R-luc construct (Figure 9) and aged for $4 \mathrm{~h}$ at $25^{\circ} \mathrm{C}$. Single embryos were homogenized in passive lysis buffer (Promega, Madison, USA); luciferase levels were quantified through luminometry using a GloMAX Multi-detection system (Promega).

\section{Supplementary material and data}

More details on experimental procedures are provided in Additional file 1. Microarray raw and preprocessed data, including probe set classification, have been submitted to the ArrayExpress database (accession numbers E-MEXP-2580 and E-MEXP-2746).

\section{Additional material}

Additional file 1: Supplemental materials and methods,

Supplemental Figures 1 to 9 and Supplemental Tables 1 to 6 .

\begin{abstract}
Abbreviations
AEL: after egg laying; ARE: AU-rich element; F-luc: firefly-luciferase; GO: Gene Ontology; miRNA/miR: microRNA; OR: Oregon Red; RBP: RNA binding protein; RMA: robust multichip average; UTR: untranslated region.
\end{abstract}

\section{Acknowledgements}

The authors wish to thank Stephen Cohen for fly stocks, Luc Berthouze, Michael Akam and Tassos Pavlopoulos for discussions, and Michelle West for sharing equipment. We also thank Pawel Herzyk and Jing Wang for expert technical advice and assistance on microarray experiments, Stijn van Dongen for support and advice on Sylamer software and Joao Osorio, Pedro Patraquim and Tracey Brazier for essential assistance during the development of some of the experiments in this study. This work was supported by the German Research Foundation (DFG; fellowship to ST), the European Union's Marie Curie Network 'Chromatin plasticity' (fellowship to $\mathrm{SA}$ ), the European Molecular Biology Organisation (WH), the MRC Laboratory of Molecular Biology as well as the Cambridge Commonwealth Trust (SCJ) and a BBSRC's Investigating Gene Function (IGF) initiative grant to CRA. The authors also wish to thank two anonymous referees for their constructive criticisms.

\section{Author details}

'John Maynard Smith Building, School of Life Sciences, University of Sussex, Falmer, Brighton, BN1 9QG, UK. ²Department of Zoology, University of Cambridge, Downing Street, Cambridge CB2 3EJ, UK. ${ }^{3}$ European Bioinformatics Institute (EBI), Wellcome Trust Genome Campus, Hinxton, Cambridge, CB10 1SD, UK. ${ }^{4}$ European Molecular Biology Laboratory (EMBL), Heidelberg, Meyerhofstraße 1, 69117 Heidelberg, Germany. ${ }^{5}$ MRC Laboratory of Molecular Biology, (LMB-MRC) Hills Road, Cambridge CB2 0QH, UK. ${ }^{6}$ Institute for Genomic Biology, University of Illinois at Urbana-Champaign, 1206 W. Gregory Drive, Urbana, IL, 61801, USA.

\section{Authors' contributions}

ST and CRA designed this study; ST carried out the experiments; WH, SA, ST, SCJ and CRA analyzed the data; ST and CRA wrote the manuscript.

\section{Competing interests}

The authors declare that they have no competing interests.

Received: 6 August 2010 Revised: 8 September 2010

Accepted: 21 September 2010 Published: 21 September 2010

\section{References}

1. Ross J: mRNA stability in mammalian cells. Microbiol Rev 1995, 59:423-450.

2. Ross J: Control of messenger RNA stability in higher eukaryotes. Trends Genet 1996, 12:171-175

3. Sorenson CM, Hart PA, Ross J: Analysis of herpes simplex virus-induced mRNA destabilizing activity using an in vitro mRNA decay system. Nucleic Acids Res 1991, 19:4459-4465.

4. Thomson AM, Rogers JT, Leedman PJ: Iron-regulatory proteins, ironresponsive elements and ferritin mRNA translation. Int J Biochem Cell Biol 1999, 31:1139-1152

5. Casey JL, Hentze MW, Koeller DM, Caughman SW, Rouault TA, Klausner RD, Harford JB: Iron-responsive elements: regulatory RNA sequences that control mRNA levels and translation. Science 1988, 240:924-928.

6. Heintz N, Sive HL, Roeder RG: Regulation of human histone gene expression: kinetics of accumulation and changes in the rate of synthesis and in the half-lives of individual histone mRNAs during the HeLa cell cycle. Mol Cell Biol 1983, 3:539-550.

7. Morris TD, Weber LA, Hickey E, Stein GS, Stein JL: Changes in the stability of a human $\mathrm{H} 3$ histone mRNA during the HeLa cell cycle. Mol Cell Biol 1991, 11:544-553.

8. Jaeck HM, Wabl M: Immunoglobulin mRNA stability varies during B lymphocyte differentiation. EMBO J 1988, 7:1041-1046.

9. Krowczynska A, Yenofsky R, Brawerman G: Regulation of messenger RNA stability in mouse erythroleukemia cells. J Mol Biol 1985, 181:231-239.

10. Gatfield D, Izaurralde E: Nonsense-mediated messenger RNA decay is initiated by endonucleolytic cleavage in Drosophila. Nature 2004, 429:575-578.

11. Gatfield D, Unterholzner L, Ciccarelli FD, Bork P, Izaurralde E: Nonsensemediated mRNA decay in Drosophila: at the intersection of the yeast and mammalian pathways. EMBO J 2003, 22:3960-3970.

12. Wang YL, Liu CL, Storey JD, Tibshirani RJ, Herschlag D, Brown PO: Precision and functional specificity in mRNA decay. Proc Natl Acad Sci USA 2002, 99:5860-5865.

13. Yang E, van Nimwegen E, Zavolan M, Rajewsky N, Schroeder M, Magnasco M, Darnell JE: Decay rates of human mRNAs: Correlation with functional characteristics and sequence attributes. Genome Res 2003, 13:1863-1872

14. Arking R, Parente A: Effects of RNA inhibitors on the development of Drosophila embryos permeabilized by a new technique. J Exp Zool 1980, 212:183-194.

15. Parente A, Arking $R$, Kalata K: Effect of DNA inhibitors upon DNA synthesis and development of Drosophila embryos. J Exp Zool 1980, 212:195-204.

16. Grigull J, Mnaimneh S, Pootoolal J, Robinson MD, Hughes TR: Genomewide analysis of mRNA stability using transcription inhibitors and microarrays reveals posttranscriptional control of ribosome biogenesis factors. Mol Cell Biol 2004, 24:5534-5547.

17. Limbourg B, Zalokar M: Permeabilization of Drosophila eggs. Dev Biol 1973, 35:382-387.

18. Mahowald AP, Kambysellis MP: Oogenesis. Genet Biol Drosophila 1980, 2:141-224.

19. Edgar BA, Datar SA: Zygotic degradation of two maternal Cdc25 mRNAs terminates Drosophila's early cell cycle program. Gene Dev 1996, 10:1966-1977.

20. Edgar BA, Kiehle CP, Schubiger G: Cell cycle control by the nucleocytoplasmic ratio in early Drosophila development. Cell 1986, 44:365-372. 
21. Edgar BA, Schubiger G: Parameters controlling transcriptional activation during early Drosophila development. Cell 1986, 44:871-877.

22. Bashirullah A, Halsell SR, Cooperstock RL, Kloc M, Karaiskakis A, Fisher WW, Fu W, Hamilton JK, Etkin LD, Lipshitz HD: Joint action of two RNA degradation pathways controls the timing of maternal transcript elimination at the midblastula transition in Drosophila melanogaster. EMBO J 1999, 18:2610-2620.

23. Semotok JL, Westwood JT, Goldman AL, Cooperstock RL, Lipshitz HD: Measuring mRNA stability during early Drosophila embryogenesis. Methods Enzymol 2008, 448:299-334.

24. Heifetz $Y, Y u J$, Wolfner MF: Ovulation triggers activation of Drosophila oocytes. Dev Biol 2001, 234:416-424.

25. Horner VL, Wolfner MF: Transitioning from egg to embryo: Triggers and mechanisms of egg activation. Dev Dyn 2008, 237:527-544.

26. Doane WW: Completion of meiosis in uninseminated eggs of Drosophila melanogaster. Science 1960, 132:677-678.

27. Tadros W, Lipshitz HD: The maternal-to-zygotic transition: a play in two acts. Development 2009, 136:3033-3042.

28. Benoit B, He CH, Zhang F, Votruba SM, Tadros W, Westwood JT, Smibert CA, Lipshitz HD, Theurkauf WE: An essential role for the RNAbinding protein Smaug during the Drosophila maternal-to-zygotic transition. Development 2009, 136:923-932.

29. Horner VL: Genetic, physiological, and proteomic analysis of egg activation in Drosophila melanogaster. PhD thesis Cornell University, Molecular Biology and Genetics (MBG) 2007.

30. Zalokar M: Autoradiographic study of protein and RNA formation during early development of Drosophila eggs. Dev Biol 1976, 49:425-437.

31. Driever W, Nüsslein-Volhard C: A gradient of bicoid protein in Drosophila embryos. Cell 1988, 54:83-93.

32. Horner VL, Czank A, Jang JK, Singh N, Williams BC, Puro J, Kubli E, Hanes SD, McKim KS, Wolfner MF: The Drosophila calcipressin sarah is required for several aspects of egg activation. Curr Biol 2006, 16:1441-1446.

33. Kennerdell JR, Carthew RW: Heritable gene silencing in Drosophila using double-stranded RNA. Nat Biotechnol 2000, 18:896-898.

34. Kennerdell JR, Yamaguchi S, Carthew RW: RNAi is activated during Drosophila oocyte maturation in a manner dependent on aubergine and spindle-E. Gene Dev 2002, 16:1884-1889.

35. Bashirullah A, Cooperstock RL, Lipshitz HD: Spatial and temporal control of RNA stability. Proc Natl Acad Sci USA 2001, 98:7025-7028.

36. Ding D, Parkhurst SM, Halsell SR, Lipshitz HD: Dynamic Hsp83 RNA localization during Drosophila oogenesis and embryogenesis. Mol Cell Biol 1993, 13:3773-3781.

37. Lipshitz HD, Smibert CA: Mechanisms of RNA localization and translational regulation. Curr Opin Genet Dev 2000, 10:476-488

38. Semotok JL, Cooperstock RL, Pinder BD, Vari HK, Lipshitz HD, Smibert CA: Smaug recruits the CCR4/POP2/NOT deadenylase complex to trigger maternal transcript localization in the early Drosophila embryo. Curr Biol 2005, 15:284-294.

39. Tadros W, Houston SA, Bashirullah A, Cooperstock RL, Semotok JL, Reed BH, Lipshitz HD: Regulation of maternal transcript destabilization during egg activation in Drosophila. Genetics 2003, 164:989-1001.

40. Semotok JL, Lipshitz HD: Regulation and function of maternal mRNA destabilization during early Drosophila development. Differentiation 2007, 75:482-506.

41. Tadros W, Lipshitz HD: Setting the stage for development: mRNA translation and stability during oocyte maturation and egg activation in Drosophila. Dev Dyn 2005, 232:593-608.

42. Semotok JL, Luo H, Cooperstock RL, Karaiskakis A, Vari HK, Smibert CA, Lipshitz HD: Drosophila maternal Hsp83 mRNA destabilization is directed by multiple SMAUG recognition elements in the open reading frame. Mol Cell Biol 2008, 28:6757-6772.

43. Tadros W, Goldman AL, Babak T, Menzies F, Vardy L, Orr-Weaver T, Hughes TR, Westwood JT, Smibert CA, Lipshitz HD: SMAUG is a major regulator of maternal mRNA destabilization in Drosophila and its translation is activated by the PAN GU kinase. Dev Cell 2007, 12:143-155.

44. Mahowald AP, Goralski TJ, Caulton JH: In vitro activation of Drosophila eggs. Dev Biol 1983, 98:437-445

45. Page AW, Orr-Weaver TL: Activation of the meiotic divisions in Drosophila oocytes. Dev Biol 1997, 183:195-207.
46. De Renzis S, Elemento O, Tavazoie S, Wieschaus EF: Unmasking activation of the zygotic genome using chromosomal deletions in the Drosophila embryo. PLOS Biol 2007, 5:e117.

47. Bornemann DJ, Park S, Phin S, Warrior R: A translational block to HSPG synthesis permits BMP signaling in the early Drosophila embryo. Development 2008, 135:1039-1047.

48. Stevens NR, Dobbelaere J, Brunk K, Franz A, Raff JW: Drosophila Ana2 is a conserved centriole duplication factor. J Cell Biol 2010, 188:313-323.

49. Sackton $\mathrm{KL}$, Lopez $\mathrm{JM}$, Berman $\mathrm{CL}$, Wolfner MF: YA is needed for proper nuclear organization to transition between meiosis and mitosis in Drosophila. BMC Dev Biol 2009, 9:43.

50. Hooper SD, Boué S, Krause R, Jensen LJ, Mason CE, Ghanim M, White KP, Furlong EE, Bork P: Identification of tightly regulated groups of genes during Drosophila melanogaster embryogenesis. Mol Syst Biol 2007, 3:72.

51. Arbeitman MN, Furlong EEM, Imam F, Johnson E, Null BH, Baker BS, Krasnow MA, Scott MP, Davis RW, White KP: Gene expression during the life cycle of Drosophila melanogaster. Science 2002, 297:2270-2275.

52. Anderson KV, Lengyel JA: Changing rates of histone mRNA synthesis and turnover in Drosophila embryos. Cell 1980, 21:717-727.

53. Anderson KV, Lengyel JA: Rates of synthesis of major classes of RNA in Drosophila embryos. Dev Biol 1979, 70:217-231.

54. Anderson KV, Lengyel JA: Changing rates of DNA and RNA synthesis in Drosophila embryos. Dev Biol 1981, 82:127-138.

55. Surdej $P$, Jacobs-Lorena M: Developmental regulation of bicoid mRNA stability is mediated by the first 43 nucleotides of the $3^{\prime}$ untranslated region. Mol Cell Biol 1998, 18:2892-2900.

56. Tadros W, Westwood JT, Lipshitz HD: The mother-to-child transition. Dev Cell 2007, 12:847-849.

57. Lam LT, Pickeral OK, Peng AC, Rosenwald A, Hurt EM, Giltnane JM, Averett LM, Zhao H, Davis RE, Sathyamoorthy M: Genomic-scale measurement of mRNA turnover and the mechanisms of action of the anti-cancer drug flavopiridol. Genome Biol 2001, 2:RESEARCH0041.

58. Narsai R, Howell KA, Millar AH, OToole N, Small I, Whelan J: Genome-wide analysis of mRNA decay rates and their determinants in Arabidopsis thaliana. Plant Cell 2007, 19:3418-3436.

59. Pilot F, Philippe JM, Lemmers C, Chauvin JP, Lecuit T: Developmental control of nuclear morphogenesis and anchoring by charleston, identified in a functional genomic screen of Drosophila cellularisation. Development 2006, 133:711-723.

60. Selinger DW, Saxena RM, Cheung KJ, Church GM, Rosenow C: Global RNA half-life analysis in Escherichia coli reveals positional patterns of transcript degradation. Genome Res 2003, 13:216-223.

61. Raghavan A, Bohjanen PR: Microarray-based analyses of mRNA decay in the regulation of mammalian gene expression. Brief Funct Genomic Proteomic 2004, 3:112-124.

62. Raghavan A, Ogilvie RL, Reilly C, Abelson ML, Raghavan S, Vasdewani J, Krathwohl M, Bohjanen PR: Genome-wide analysis of mRNA decay in resting and activated primary human T lymphocytes. Nucleic Acids Res 2002, 30:5529-5538.

63. He F, Li X, Spatrick P, Casillo R, Dong S, Jacobson A: Genome-wide analysis of mRNAs regulated by the nonsense-mediated and 5 to 3 mRNA decay pathways in yeast. Mol Cell 2003, 12:1439-1452.

64. Bernstein JA, Khodursky AB, Lin PH, Lin-Chao S, Cohen SN: Global analysis of mRNA decay and abundance in Escherichia coli at single-gene resolution using two-color fluorescent DNA microarrays. Proc Natl Acad Sci USA 2002, 99:9697-9702.

65. Janga SC, Babu MM: Transcript stability in the protein interaction network of Escherichia coli. Mol Biosyst 2009, 5:154-162.

66. Fly-FISH: a database of Drosophila embryo mRNA localization patterns. [http://fly-fish.ccbr.utoronto.ca/].

67. Lécuyer E, Yoshida H, Parthasarathy N, Alm C, Babak T, Cerovina T, Hughes TR, Tomancak P, Krause HM: Global analysis of mRNA localization reveals a prominent role in organizing cellular architecture and function. Cell 2007, 131:174-187.

68. Qin X, Ahn S, Speed TP, Rubin GM: Global analyses of mRNA translational control during early Drosophila embryogenesis. Genome Biol 2007, 8:R63.

69. Gouw JW, Pinkse MWH, Vos HR, Moshkin Y, Verrijzer CP, Heck AJR, Krijgsveld J: In vivo stable isotope labeling of fruit flies reveals posttranscriptional regulation in the maternal-to-zygotic transition. MCP 2009, 8:1566-1578. 
70. van Dongen S, Abreu-Goodger C, Enright AJ: Detecting microRNA binding and siRNA off-target effects from expression data. Nat Methods 2008, 5:1023-1025.

71. Cairrao F, Halees AS, Khabar KSA, Morello D, Vanzo N: AU-rich elements regulate Drosophila gene expression. Mol Cell Biol 2009, 29:2636-2643.

72. Bushati N, Stark A, Brennecke J, Cohen SM: Temporal reciprocity of miRNAs and their targets during the maternal-to-zygotic transition in Drosophila. Curr Biol 2008, 18:501-506.

73. Parisi M, Lin $\mathrm{H}$ : The Drosophila pumilio gene encodes two functional protein isoforms that play multiple roles in germline development, gonadogenesis, oogenesis and embryogenesis. Genetics 1999 , 153:235-250.

74. Smibert CA, Wilson JE, Kerr K, Macdonald PM: smaug protein represses translation of unlocalized nanos mRNA in the Drosophila embryo. Gene Dev 1996, 10:2600-2609.

75. Ruby JG, Stark A, Johnston WK, Kellis M, Bartel DP, Lai EC: Evolution, biogenesis, expression, and target predictions of a substantially expanded set of Drosophila microRNAs. Genome Res 2007, 17:1850-1864.

76. miRBase: the microRNA database. [http://www.mirbase.org/].

77. Griffiths-Jones S, Saini HK, Dongen S, Enright AJ: miRBase: tools for microRNA genomics. Nucleic Acids Res 2008, 36(Database):D154-158.

78. Ma J, Flemr M, Stein P, Berninger P, Malik R, Zavolan M, Svoboda P, Schultz RM: MicroRNA activity is suppressed in mouse oocytes. Curr Biol 2010, 20:265-270.

79. Suh N, Baehner L, Moltzahn F, Melton C, Shenoy A, Chen J, Blelloch R: MicroRNA function is globally suppressed in mouse oocytes and early embryos. Curr Biol 2010, 20:271-277.

80. Gamberi C, Johnstone O, Lasko P: Drosophila RNA binding proteins. Int Rev Cytol 2006, 248:43-139.

81. Mittal N, Roy N, Babu MM, Janga SC: Dissecting the expression dynamics of RNA-binding proteins in posttranscriptional regulatory networks. Proc Natl Acad Sci USA 2009, 106:20300-20305.

82. FlyBase: A Database of Drosophila Genes \& Genomes. [http://flybase.org/].

83. Mahone M, Saffman EE, Lasko PF: Localized Bicaudal-C RNA encodes a protein containing a $\mathrm{KH}$ domain, the RNA binding motif of FMR1. EMBO J 1995, 14:2043-2055.

84. Wilhelm JE, Mansfield J, Hom-Booher N, Wang S, Turck CW, Hazelrigg T, Vale RD: Isolation of a ribonucleoprotein complex involved in mRNA localization in Drosophila oocytes. J Cell Biol 2000, 148:427-440.

85. Mansfield JH, Wilhelm JE, Hazelrigg T: Ypsilon Schachtel, a Drosophila Ybox protein, acts antagonistically to Orb in the oskar mRNA localization and translation pathway. Development 2002, 129:197-209.

86. Pisa V, Cozzolino M, Gargiulo S, Ottone C, Piccioni F, Monti M, Gigliotti S, Talamo F, Graziani F, Pucci P: The molecular chaperone Hsp90 is a component of the cap-binding complex and interacts with the translational repressor Cup during Drosophila oogenesis. Gene 2009, 432:67-74.

87. Chicoine J, Benoit P, Gamberi C, Paliouras M, Simonelig M, Lasko P: Bicaudal-C recruits CCR4-NOT deadenylase to target mRNAs and regulates oogenesis, cytoskeletal organization, and its own expression. Dev Cell 2007, 13:691-704

88. Snee MJ, Macdonald PM: Bicaudal C and trailer hitch have similar roles in gurken mRNA localization and cytoskeletal organization. Dev Biol 2009, 328:434-444.

89. Herold N, Will CL, Wolf E, Kastner B, Urlaub H, Luhrmann R: Conservation of the protein composition and electron microscopy structure of Drosophila melanogaster and human spliceosomal complexes. Mol Cell Biol 2009, 29:281-301.

90. Yang D, Lu H, Erickson JW: Evidence that processed small dsRNAs may mediate sequence-specific mRNA degradation during RNAi in Drosophila embryos. Curr Biol 2000, 10:1191-1200.

91. Rehwinkel J, Herold A, Gari K, Kocher T, Rode M, Ciccarelli FL, Wilm M, Izaurralde E: Genome-wide analysis of mRNAs regulated by the THO complex in Drosophila melanogaster. Nat Struct Mol Biol 2004, 11:558-566.

92. Edgar BA, O'Farrell PH: The three postblastoderm cell cycles of Drosophila embryogenesis are regulated in G2 by string. Cell 1990, 62:469-480.

93. Foe VE: Mitotic domains reveal early commitment of cells in Drosophila embryos. Development 1989, 107:1-22

94. Paillard L, Osborne HB: East of EDEN was a poly (A) tail. Biol Cell 2003, 95:211-220.
95. Wreden C, Verrotti AC, Schisa JA, Lieberfarb ME, Strickland S: Nanos and pumilio establish embryonic polarity in Drosophila by promoting posterior deadenylation of hunchback mRNA. Development 1997, 124:3015-3023.

96. Boswell RE, Mahowald AP: tudor, a gene required for assembly of the germ plasm in Drosophila melanogaster. Cell 1985, 43:97-104.

97. Xue L, Noll M: Drosophila female sexual behavior induced by sterile males showing copulation complementation. Proc Natl Acad Sci USA 2000, 97:3272-3275.

98. Xu P, Vernooy SY, Guo M, Hay BA: The Drosophila microRNA Mir-14 suppresses cell death and is required for normal fat metabolism. Curr Biol 2003, 13:790-795.

99. Theurkauf WE: Immunofluorescence analysis of the cytoskeleton during oogenesis and early embryogenesis. Methods Cell Biol 1994, 44:489-505.

100. Jacobs-Lorena M, Crippa M: Mass fractionation of Drosophila egg chambers. Dev Biol 1977, 57:385-392.

101. Mahowald AP: Mass isolation of fly tissues. Methods Cell Biol 1994, 44:129-142.

102. King RC: Ovarian Development inDrosophila melanogaster. New York and London: Academic Press 1970.

103. Core-Team: R: A language and environment for statistical computing. [http://cran.r-project.org/doc/manuals/refman.pdf].

104. Gentleman R, Carey V, Bates D, Bolstad B, Dettling M, Dudoit S, Ellis B, Gautier L, Ge Y, Gentry J: Bioconductor: open software development for computational biology and bioinformatics. Genome Biol 2004, 5:R80.

105. Huber W, von Heydebreck A, Sueltmann H, Poustka A, Vingron M Parameter estimation for the calibration and variance stabilization of microarray data. Stat Appl Genet Mol Biol 2003, 2:Article 3 [http://www. bepress.com/sagmb/vol2/iss1/art3/].

106. Huber W, von Heydebreck A, Sultmann H, Poustka A, Vingron M: Variance stabilization applied to microarray data calibration and to the quantification of differential expression. Bioinformatics 2002, 18 Suppl 1 : S96-S104.

107. Irizarry RA, Bolstad BM, Collin F, Cope LM, Hobbs B, Speed TP: Summaries of Affymetrix GeneChip probe level data. Nucleic Acids Res 2003, 31:e15.

108. Boyle El, Weng S, Gollub J, Jin H, Botstein D, Cherry JM, Sherlock G: GO* TermFinder - open source software for accessing Gene Ontology information and finding significantly enriched Gene Ontology terms associated with a list of genes. Bioinformatics 2004, 20:3710-3715.

109. Gerber AP, Luschnig S, Krasnow MA, Brown PO, Herschlag D: Genome-wide identification of mRNAs associated with the translational regulator PUMILIO in Drosophila melanogaster. Proc Natl Acad Sci USA 2006, 103:4487-4492.

110. Storey JD: A direct approach to false discovery rates. J Roy Stat Soc B Met 2002, 64:479-498.

111. Hartenstein V: Stages of Drosophila embryogenesis. In The Embryonic Development of Drosophila melanogaster. Edited by: Campos-Ortega JA, Hartenstein V. Berlin: Springer; , 2 1997:9-102.

112. MicroCosm Targets Version 5. [http://www.ebi.ac.uk/enright-srv/ microcosm/htdocs/targets/v5/]

113. Sonnenblick BP: The early embryology of Drosophila melanogaster. In Biology of Drosophila. Edited by: Demerec M. New York: Cold Spring Harbor Laboratory Press; 1950:62-167.

114. Petri WH, Wyman AR, Kafatos FC: Specific protein synthesis in cellular differentiation* $1::$ III. The eggshell proteins of Drosophila melanogaster and their program of synthesis. Dev Biol 1976, 49:185-199.

115. Parman C, Halling C, Gentleman R: affyQCReport: QC report generation for affyBatch objects. [http://www.bioconductor.org/packages/2.6/bioc/ html/affyQCReport.html]

116. Gautier L, Cope L, Bolstad BM, Irizarry RA: affy - analysis of Affymetrix GeneChip data at the probe level. Bioinformatics 2004, 20:307-315.

117. Wilson CL, Miller CJ: Simpleaffy: a BioConductor package for Affymetrix quality control and data analysis. Bioinformatics 2005, 21:3683-3685.

118. Bolstad BM: Low-level analysis of high-density oligonucleotide array data: background, normalization and summarization. PhD thesis University of California 2004.

119. Fraley C, Raftery AE: Model-based clustering, discriminant analysis, and density estimation. J Am Stat Assoc 2002, 97:611-632.

120. Gentleman R, Carey V, Huber W, Hahne F: genefilter: methods for filtering genes from microarray experiments. [http://www.bioconductor.org/ packages/2.6/bioc/html/genefilter.html]. 
121. Gentleman R: geneplotter: Graphics related functions for Bioconductor. [http://www.bioconductor.org/packages/2.6/bioc/html/geneplotter.html].

122. Brettschneider J, Collin F, Bolstad BM, Speed TP: Quality assessment for short oligonucleotide microarray data. Technometrics 2008, 50:241-264.

123. Gentleman R, Huber W, Carey VJ, Irizarry RA, Dudoit S: Bioinformatics and Computational Biology Solutions Using R and Bioconductor. New York Springer 2005

124. Kauffmann A, Gentleman R, Huber W: arrayQualityMetrics - a bioconductor package for quality assessment of microarray data. Bioinformatics 2009, 25:415-416.

125. Kauffmann A, Huber W: Microarray data quality control improves the detection of differentially expressed genes. Genomics 2010, 95:138-142.

126. Affymetrix. [http://www.affymetrix.com].

127. Bolstad B: affyPLM: Fitting probe level models. [http://www.bioconductor. org/packages/2.6/bioc/vignettes/affyPLM/inst/doc/AffyExtensions.pdf].

128. Guan SH, Bonnett L, Brettschneider J: Using gene subsets in the assessment of microarray data quality for time course experiments. Centre for Research in Statistical Methodology (CRiSM) 2009 [http://www2. warwick.ac.uk/fac/sci/statistics/crism/research/2009/paper09-24], Paper No. 09-24.

129. Guan SH, Zheng J, Brettschneider J: Microarray data quality assessment for developmental time series. In Systems Biology and Statistical Bioinformatics. Edited by: Barber S, Baxter PD, Mardia KV. Leeds: University Press; 2007:79-82.

130. GENE-E. [http://www.broadinstitute.org/cancer/software/GENE-E/]

131. Breitling R, Amtmann A, Herzyk P: Iterative Group Analysis (iGA): a simple tool to enhance sensitivity and facilitate interpretation of microarray experiments. BMC Bioinformatics 2004, 5:34.

132. Rozen S, Skaletsky H: Primer3 on the WWW for general users and for biologist programmers. Methods Mol Biol 2000, 132:365-386.

133. Newbury SF: Control of mRNA stability in eukaryotes. Biochem Soc Trans 2006, 34:30-34

134. Lackner DH, Beilharz TH, Marguerat S, Mata J, Watt S, Schubert F, Preiss T, Bähler J: A network of multiple regulatory layers shapes gene expression in fission yeast. Mol Cell 2007, 26:145-155.

135. Salles FJ, Lieberfarb ME, Wreden C, Gergen JP, Strickland S: Coordinate initiation of Drosophila development by regulated polyadenylation of maternal messenger RNAs. Science 1994, 266:1996-1999.

136. Lieberfarb ME, Chu T, Wreden C, Theurkauf W, Gergen JP, Strickland S Mutations that perturb poly(A)-dependent maternal mRNA activation block the initiation of development. Development 1996, 122:579-588.

137. Affymetrix: Eukaryotic target preparation. GeneChip Expression Analysis Technical Manual Affymetrix 2007, 2.1.5-2.1.22.

138. Steller H, Pirrotta V: Regulated expression of genes injected into early Drosophila embryos. EMBO J 1984, 3:165.

139. Primer3: Pick primers from a DNA sequence. [http://frodo.wi.mit.edu/ primer3/].

doi:10.1186/gb-2010-11-9-r93

Cite this article as: Thomsen et al.: Genome-wide analysis of mRNA decay patterns during early Drosophila development. Genome Biology 2010 11:R93.

\section{Submit your next manuscript to BioMed Central and take full advantage of:}

- Convenient online submission

- Thorough peer review

- No space constraints or color figure charges

- Immediate publication on acceptance

- Inclusion in PubMed, CAS, Scopus and Google Scholar

- Research which is freely available for redistribution

Submit your manuscript at www.biomedcentral.com/submit 